ARTICLE

\title{
PKA drives an increase in AMPA receptor unitary conductance during LTP in the hippocampus
}

Pojeong Park1,2,3,4, John Georgiou (10 3, Thomas M. Sanderson (1,2,3, Kwang-Hee Ko², Heather Kang 1,2,3,4,

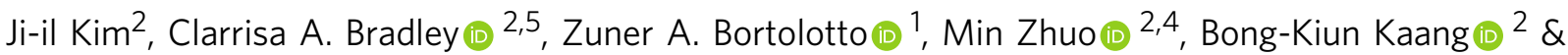
Graham L. Collingridge (1,2,3,4,6凶

Long-term potentiation (LTP) at hippocampal CA1 synapses can be expressed by an increase either in the number $(\mathrm{N})$ of AMPA ( $\alpha$-amino-3-hydroxy-5-methyl-4-isoxazole propionic acid) receptors or in their single channel conductance $(\gamma)$. Here, we have established how these distinct synaptic processes contribute to the expression of LTP in hippocampal slices obtained from young adult rodents. LTP induced by compressed theta burst stimulation (TBS), with a $10 \mathrm{~s}$ inter-episode interval, involves purely an increase in $N\left(L T P_{N}\right)$. In contrast, either a spaced TBS, with a 10 min inter-episode interval, or a single TBS, delivered when PKA is activated, results in LTP that is associated with a transient increase in $\gamma\left(\right.$ LTP $\left._{\gamma}\right)$, caused by the insertion of calcium-permeable (CP)-AMPA receptors. Activation of CaMKII is necessary and sufficient for $L T P_{N}$ whilst PKA is additionally required for $L T P_{\gamma}$. Thus, two mechanistically distinct forms of LTP co-exist at these synapses.

\footnotetext{
${ }^{1}$ Glutamate Receptor Group, School of Physiology, Pharmacology and Neuroscience, University of Bristol, Bristol, United Kingdom. ${ }^{2}$ Department of Biological Sciences, College of Natural Sciences, Seoul National University, Seoul 08826, Korea. ${ }^{3}$ Lunenfeld-Tanenbaum Research Institute, Mount Sinai Hospital, Toronto, ON M5G 1X5, Canada. ${ }^{4}$ Department of Physiology, University of Toronto, Toronto, ON M5S 1A8, Canada. ${ }^{5}$ Neurosciences and Mental Health, Peter Gilgan Centre for Research and Learning, The Hospital for Sick Children, Toronto, ON M5G OA4, Canada. ${ }^{6}$ TANZ Centre for Research in Neurodegenerative Diseases, University of Toronto, Toronto, ON M5S 1A8, Canada. ${ }^{\circledR}$ email: collingridge@lunenfeld.ca
} 
ong-term potentiation (LTP) of synaptic function is considered the major process underlying learning and memory ${ }^{1}$ where it is involved in synaptic engram formation ${ }^{2,3}$, yet the underlying cellular mechanisms remain incompletely understood. The best-characterized form of LTP occurs at the Schaffer collateral-commissural pathway (SCCP) in the hippocampus, where it is triggered by synaptic activation of NMDA ( $N$-methyl$\mathrm{D}$-aspartate) receptors ${ }^{4}$ and is expressed as a persistent increase in AMPA ( $\alpha$-amino-3-hydroxy-5-methyl-4-isoxazole propionic acid) receptor-mediated synaptic transmission ${ }^{5}$. This modification is primarily due to a functional modulation of AMPA receptors (AMPARs), which may involve a change in the number of active channels $(N)$ (termed $\mathrm{LTP}_{\mathrm{N}}$ ) and/or their single-channel conductance $(\gamma)$ properties (termed $\left.\operatorname{LTP}_{\gamma}\right)\left(\right.$ e.g. $\left.{ }^{6-9}\right)$. Whilst there is considerable evidence that $\mathrm{LTP}_{\mathrm{N}}$ is triggered by activation of $\mathrm{Ca}^{2+} /$ calmodulin-dependent kinase II (CaMKII) ${ }^{10,11}$ and involves exocytosis and lateral diffusion of AMPARs ${ }^{12,13}$, the mechanisms underlying $\mathrm{LTP}_{\gamma}$ are largely unknown. The two most likely molecular mechanisms involve (i) CaMKII-mediated phosphorylation of Ser831 of GluA1, which can result in an increase in the time AMPARs dwell in higher conductance states $^{14-16}$ or (ii) the insertion of calcium-permeable AMPA receptors (CP-AMPARs), which have a higher $\gamma$ than their calcium-impermeable $(\mathrm{CI})$ counterparts ${ }^{17,18}$.

In the present study, we have tested the hypothesis that $\mathrm{LTP}_{\gamma}$ is due to the insertion of CP-AMPARs in young adult rodents using two theta burst stimulation (TBS) induction protocols that differed only in the timing between episodes, and applied peakscaled non-stationary fluctuation analysis (NSFA) ${ }^{19-21}$ to estimate $\gamma$ before and after the induction of LTP6,15,22-25. We found that the compressed TBS protocol (cTBS - inter-episode interval of $10 \mathrm{~s}$ ) resulted exclusively in $\mathrm{LTP}_{\mathrm{N}}$, for which CaMKII was both necessary and sufficient. In contrast, a spaced TBS protocol (sTBS - inter-episode interval of $10 \mathrm{~min}$ ) resulted in a transient increase in $\gamma$, lasting $\sim 15 \mathrm{~min}$, which was due to the insertion of CPAMPARs and required both CaMKII and PKA. Insertion of CPAMPARs mediates both the initial expression of $\mathrm{LTP}_{\gamma}$, by enhancing the net synaptic unitary conductance, and helps trigger the processes that lead to a persistent increase in synaptic efficacy that outlasts the increase in $\gamma$. Since the PKA-dependent form of LTP also requires de novo protein synthesis and has stimulation features similar to spaced behavioural learning, $\mathrm{LTP}_{\gamma}$ is likely to underlie the formation of synaptic engrams and long-term memory.

\section{Results}

An increase in $\gamma$ is specifically triggered by a sTBS protocol. Simultaneous field excitatory postsynaptic potential (fEPSP) recordings from stratum radiatum and somatic whole-cell recordings were obtained in response to baseline stimulation of two independent SCCP inputs (Fig. 1a). TBS was delivered to one input (test), while the second input served as a control for stability and heterosynaptic effects (Fig. 1c, d). Synaptic potentiation was quantified and $\gamma$ was estimated using NSFA (Fig. 1e, f), as described previously ${ }^{6}$. To optimize the estimates of $\gamma$ we used minimal stimulation and restricted our measurements to the first 20-30 min following TBS, since $\gamma$ estimates are extremely sensitive to small fluctuations in series resistance ${ }^{20}$. Thus, our study focused on the induction and initial expression mechanisms of LTP.

In the first series of experiments we delivered three episodes of TBS, with each episode comprising 5 shocks at $100 \mathrm{~Hz}$ delivered 5 times at $5 \mathrm{~Hz}$ (i.e. 75 stimuli in total; see Fig. 1b schematic); in interleaved experiments we either delivered these three episodes as a cTBS (10 s inter-episode interval) or as a sTBS (10 min inter- episode interval). We referred to the resultant potentiation as cLTP (Fig. 2a-i) and sLTP (Fig. 2j-r), respectively. In response to cTBS there was a substantial cLTP (Fig. 2a), with EPSC amplitudes increasing to $212 \pm 11 \%$ of baseline, averaged over the first $10 \mathrm{~min}$ after induction (Fig. 2b). For 22 neurons from 15 rats $(n=22 / 15)$, we obtained $\gamma$ estimates in $10 \mathrm{~min}$ epochs and found it to be unaltered throughout (Fig. $2 \mathrm{c}-\mathrm{g}$ ). The $\gamma$ values were $5.1 \pm 0.3 \mathrm{pS}$, (baseline), $5.3 \pm 0.4 \mathrm{pS}$ (first $10 \mathrm{~min}$ epoch post cTBS; $\mathrm{LTP}_{10} ; t_{21}=1.23, p=0.2327$, vs baseline, paired Student's $t$ test) and $5.2 \pm 0.4 \mathrm{pS}$ (second $10 \mathrm{~min}$ epoch post cTBS; $\mathrm{LTP}_{20} ; t_{21}=$ $0.33, p=0.7452$; Fig. 2d). The control input was also stable throughout $(4.9 \pm 0.4 \mathrm{pS}, 4.5 \pm 0.3 \mathrm{pS}$ and $4.8 \pm 0.3 \mathrm{pS}$ at the corresponding time-points; Fig. 2d). The lack of change in $\gamma$ was also clearly evident in the plots from individual experiments for control (Fig. 2e) and test inputs (Fig. 2f) and in the cumulative distribution plots (Fig. $2 \mathrm{~g}$ ). The lack of change in $\gamma$ was observed over a wide range of cLTP magnitudes (Fig. $2 \mathrm{~h}$ ).

In response to sTBS the results were strikingly different. For this set of experiments, whole-cell recordings were obtained shortly after delivery of the second TBS episode and the effects of the third TBS were evaluated (Fig. 2j). This method was necessary because of the rapid wash-out of LTP with low access whole-cell recordings. In response to the third TBS there was a substantial additional LTP, with EPSC amplitudes increasing to $177 \pm 9 \%$ of baseline, averaged over the first $10 \mathrm{~min}$ after induction (Fig. $2 \mathrm{k}$ ). The estimate of $\gamma$ upon break in was significantly higher $(6.9 \pm 0.4$ $\mathrm{pS})$ compared to the control input $\left(4.9 \pm 0.4 \mathrm{pS}\right.$; Fig. $2 \mathrm{n}-\mathrm{O} ; t_{22}=$ 3.22, $p=0.0039$, paired Student's $t$ test) and this was further increased in response to the third episode of TBS to $8.4 \pm 0.4 \mathrm{pS}$ $\left(\mathrm{LTP}_{10} ; t_{22}=3.75, p=0.0011\right.$, Fig. $\left.2 \mathrm{l}, \mathrm{m}, \mathrm{o}, \mathrm{p} ; n=23 / 17\right)$. However, when we quantified $\gamma$ at 10-20 min after the last TBS, the value $(5.5 \pm 0.3 \mathrm{pS})$ was no longer significantly different from the control input $\left(\mathrm{LTP}_{20} ; t_{22}=2.01, p=0.0570\right.$, paired Student's $t$ test; Fig. $2 \mathrm{~m}$ ). In contrast to the test input, sTBS did not result in a significant $\gamma$ change in the control input $(4.9 \pm 0.4 \mathrm{pS}, 5.4 \pm 0.4 \mathrm{pS}$ and $4.6 \pm 0.3 \mathrm{pS}$ at the corresponding time points; Fig. $2 \mathrm{~m}, \mathrm{n}$ ). Thus, the increase in $\gamma$ is specifically related to sLTP. Furthermore, this increase in $\gamma$ positively correlated with the magnitude of sLTP (Fig. 2q).

Since sLTP, but not cLTP, is associated with the insertion of CP-AMPARs ${ }^{26,27}$ these results suggest that CP-AMPARs may account for the increase in $\gamma$. CP-AMPARs have slightly faster decay kinetics $\left(\tau_{\text {decay }}\right)$ than CI-AMPARs ${ }^{25,28}$, which can be detected using single exponential fits to EPSC decays. We found that cLTP was not associated with an alteration in $\tau_{\text {decay }}$ (Fig. $2 \mathrm{i}$, Supplementary Table $1 ; t_{21}=0.66, p=0.5146$, paired Student's $t$ test), whereas sLTP was associated with a highly significant decrease in $\tau_{\text {decay }}$ (Supplementary Table $1 ; p=0.0051, t_{22}=3.11$, paired Student's $t$ test). A regression analysis showed a trend for the $\tau_{\text {decay }}$ to be inversely related with the increase in $\gamma$ (Fig. 2 r; $p=$ $\left.0.0712, F_{(1,21)}=3.61\right)$. Therefore, the kinetic analysis provides additional support for the notion that insertion of CP-AMPARs occurs during the induction of LTP in response to a sTBS.

The role of PKA in LTP $_{\gamma}$. It is established that elevating cAMP by, for example, use of the phosphodiesterase 4 inhibitor rolipram, enables a weak stimulus to generate an enhanced PKAdependent form of $\mathrm{LTP}^{29}$. Previously, we found that in the presence of rolipram a weak TBS, comprising one episode of TBS, generated an LTP that is largely dependent on the insertion of CP-AMPARs ${ }^{26}$. Here we used this same method as an independent means to investigate whether insertion of CP-AMPARs are responsible for the increase in $\gamma$. Since only one TBS is required to induce the PKA-dependent form of LTP in the presence of rolipram we could make $\gamma$ measurements before and after the full 


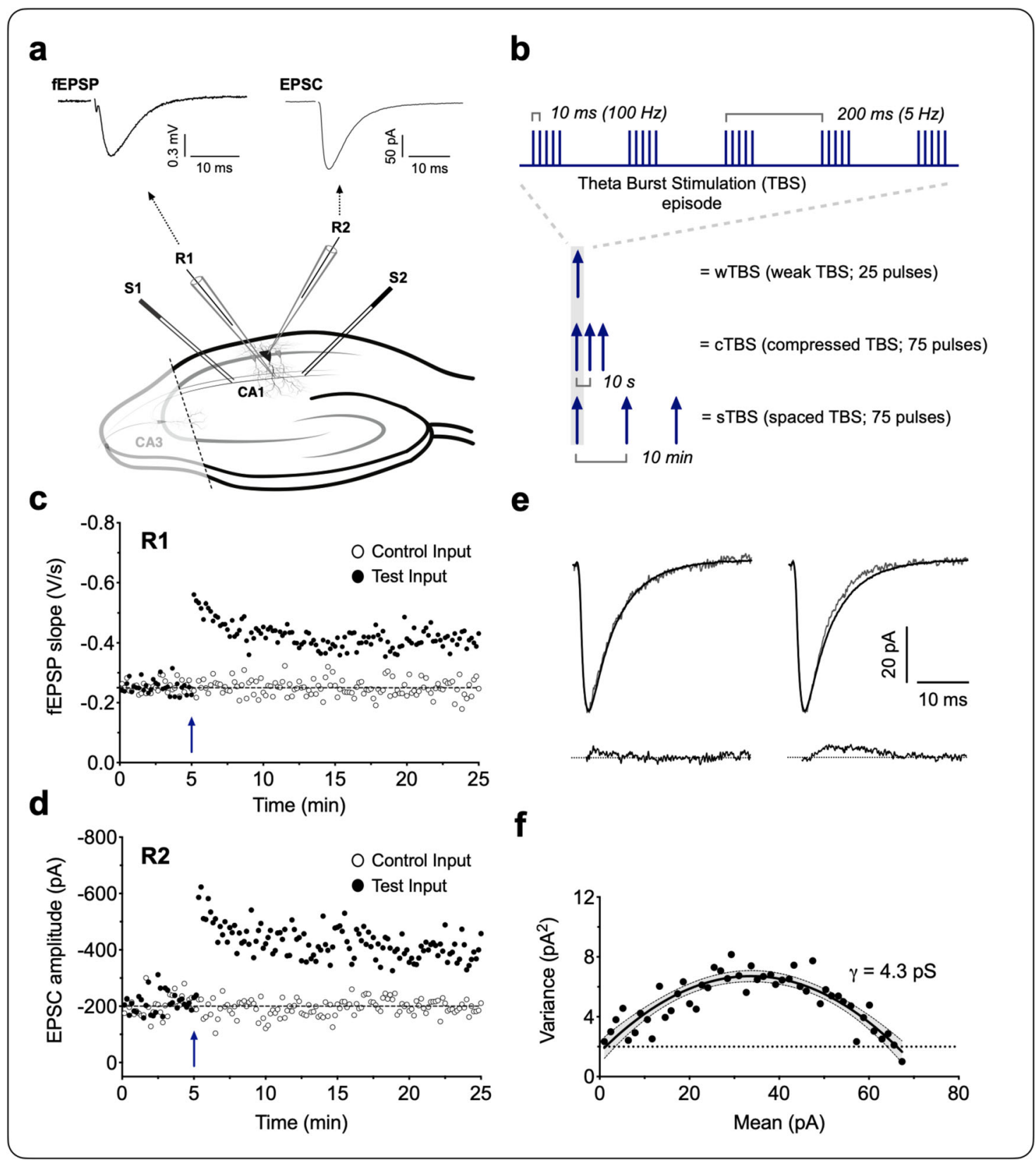

Fig. 1 LTP and non-stationary fluctuation analysis (NSFA) methodology. a Schematic of a hippocampal brain slice for LTP experiments, and the positioning of recording (R1, R2) and stimulating (S1, S2) electrodes. The CA3 region was cut (dashed line) to reduce neuronal excitability. Representative field and whole cell responses (fEPSP and EPSC), simultaneously obtained from CA1 neurons. Five consecutive responses were averaged and the stimulus artifacts were blanked for clarity. $\mathbf{b}$ Induction protocols for weak, compressed and spaced TBS (WTBS, cTBS and sTBS) are graphically summarized. c Representative fEPSP recordings for LTP evoked by a single episode of TBS (weak TBS, blue arrow). d Simultaneously obtained EPSC recordings. e Upper traces are two sets of representative waveforms for individual sweeps (thin lines), superimposed with the scaled mean of 57 EPSCs (thick lines). Lower traces are the subtraction of the scaled mean from the representative individual EPSCs. $\mathbf{f}$ Corresponding current-variance relationship to estimate the unitary conductance $(\gamma)$. Fluctuation of the individual decays was plotted against the mean EPSC. Solid line is a parabolic fit with $95 \%$ confidence intervals (shaded). Dotted line, the background average variance.

induction of LTP. As illustrated in Fig. 3a, b, a single episode of TBS (wTBS; comprising 25 stimuli), when delivered in the presence of rolipram $(1 \mu \mathrm{M})$, generated a robust LTP $(234 \pm 14 \%$ of baseline for test vs. $121 \pm 6 \%$ for control input). We found that this LTP was also associated with a transient increase in $\gamma$ (baseline $=4.9 \pm 0.4 \mathrm{pS}, \quad \mathrm{LTP}_{10^{\prime}}=8.0 \pm 0.6 \mathrm{pS} ; \quad t_{20}=5.90, \quad p<$ $0.0001)$ that returned to baseline by the second $10 \mathrm{~min}$ epoch $\left(\mathrm{LTP}_{20^{\prime}}=5.4 \pm 0.3 \mathrm{pS} ; t_{20}=1.39, p=0.1810\right.$, paired Student's $t$ test) following the wTBS $(n=21 / 15$; Fig. $3 \mathrm{c}, \mathrm{d}, \mathrm{f}, \mathrm{g})$. This potentiation required the wTBS since the control input was largely unaffected $(5.1 \pm 0.3 \mathrm{pS}, 5.4 \pm 0.5 \mathrm{pS}$ and $4.8 \pm 0.3 \mathrm{pS}$ at the corresponding time points; Fig. $3 \mathrm{~d}$, e) and since the baseline $\gamma$ values in the presence of rolipram were not significantly different to the baseline $\gamma$ values in its absence (Fig. 3d-f; Supplementary Table 1). As was the case with the sLTP, the size of the change in $\gamma$ correlated with the magnitude of LTP $\left(p=0.0024, F_{(1,19)}=\right.$ 12.27; Fig. 3h). Additionally, there was an associated reduction in $\tau_{\text {decay }}\left(p=0.0007, t_{20}=3.99\right.$, paired Student's $t$ test; Supplementary Table 1) that also negatively correlated with the increased $\gamma\left(p=0.0199, F_{(1,19)}=6.46\right.$; Fig. 3i). These results 


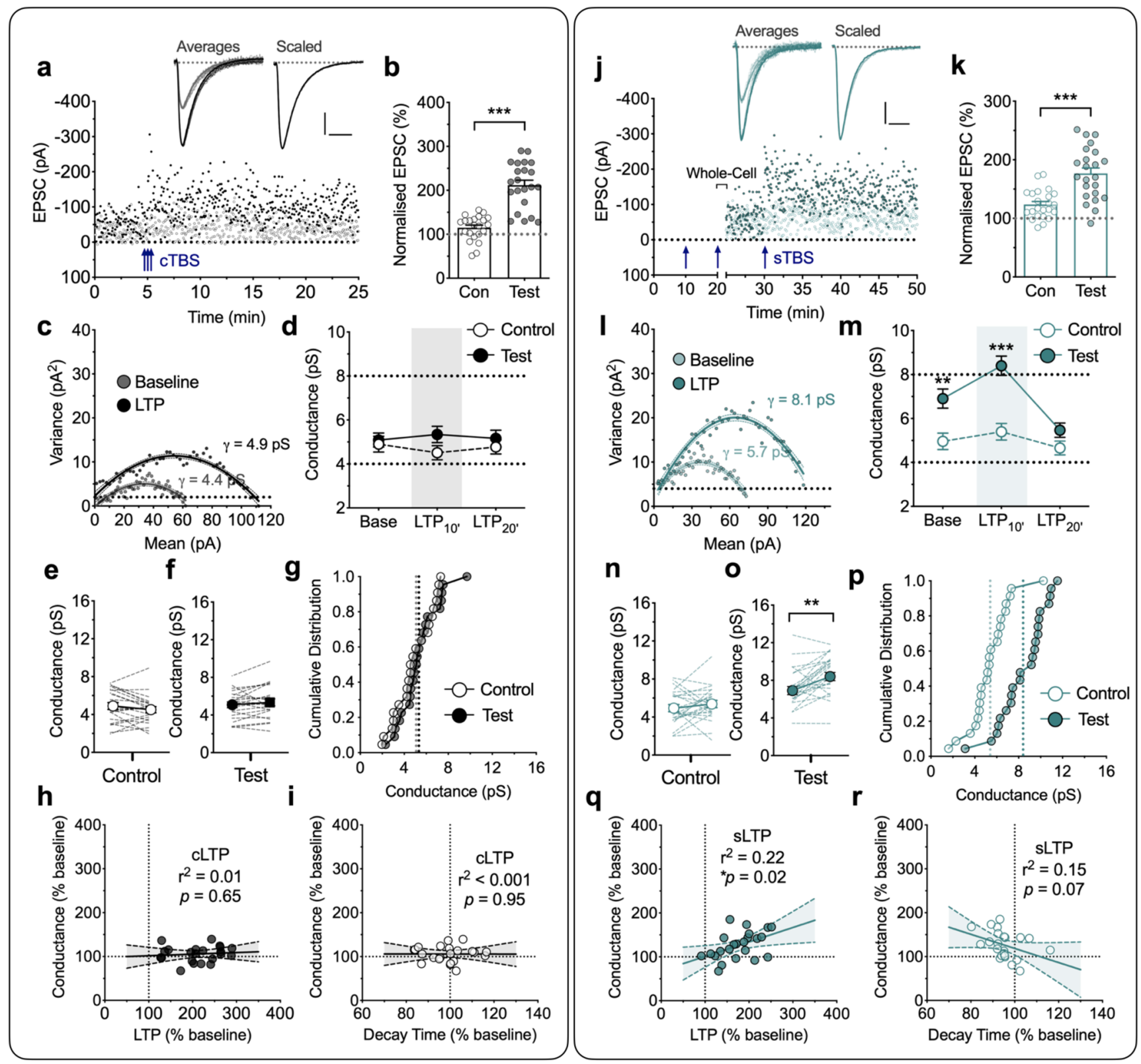

Fig. 2 Increased AMPA receptor unitary conductance $(\boldsymbol{\gamma})$ during sLTP, but not cLTP. a A representative LTP experiment with sample traces for baseline and post TBS - the mean of selected records for analysis, superimposed with peak-scaled individual traces (10 successive sweeps, thin lines; baseline = grey, LTP $=$ black). Scaled trace is from the baseline normalized to the LTP. Scale bars: 20 pA and $10 \mathrm{~ms}$. Two inputs were stimulated alternately and cTBS ( $3 \mathrm{x}$ TBS with an inter-episode interval of $10 \mathrm{~s}$; blue arrows) delivered to one input (filled symbols) with the second input (open symbols) serving as a control (Con). b Levels of cTBS-induced LTP (cLTP) for control and test inputs, quantified during the 10 min epoch after the induction (mean \pm SEM, $n=22$ neurons from 15 animals; $t_{21}=8.545, p<0.0001$, two-sided paired Student's $t$ test). c Corresponding current-variance relationship of the EPSCs for the test input. The unitary channel conductance $(\gamma)$ of AMPA receptors was estimated during baseline (grey) and after the induction of LTP (LTP 10 ; ; black). d Grouped comparison of control and test input $\gamma$ estimates for baseline and the initial $10 \mathrm{~min}$ epoch $\left(\mathrm{LTP}_{10^{\prime}}\right)$ and the subsequent $10 \mathrm{~min}$ epoch $\left(\mathrm{LTP}_{20^{\prime}}\right) . n=22 \mathrm{neurons}$ from 15 animals. e, f Summary plot for the $\gamma$ at baseline (left) and LTP $10^{\prime}$ (right) for control (e) and test (f) inputs. Individual values from each neuron are connected by lines. Circles indicate mean values. $\mathbf{g}$ Cumulative distribution of the same data set for $\mathrm{LTP}_{10}$. Dotted lines indicate the mean values for each input. $\mathbf{h}$, $\mathbf{i}$ Analysis of the relationships of $\gamma$ with $\operatorname{LTP}\left(p=0.6517, F_{(1,20)}=0.2101, F\right.$-test $)(\mathbf{h})$ and EPSC decay time $\left(p=0.9521, F_{(1,20)}=0.0037, F\right.$-test $)(\mathbf{i})$. Linear regression with $95 \%$ confidence intervals (shaded) for the amount of CLTP and the corresponding level of $\gamma$. j-r Equivalent analysis for the LTP induced by sTBS ( $3 \times$ TBS at inter-episode interval of $10 \mathrm{~min}$; see arrows). The whole-cell recordings were obtained after the second TBS. This was necessary due to the lability of LTP washout. $\mathbf{k}$ Levels of sTBS-induced LTP (sLTP) for control and test inputs, quantified during the 10 min epoch after the induction ( $n=23$ neurons from 17 animals; $t_{22}=5.238, p<0.0001$, two-sided paired Student's $t$ test). $\mathbf{m}$-o Statistical analysis between control and test pathways $\left(t_{22}=3.220, p=0.0039\right.$ for baseline and $t_{22}=6.123, p<0.0001$ for LTP $_{10}$, two-sided paired Student's $t$ test) ( $\left.\mathbf{m}\right)$ and within pathway analysis for control $\left(t_{22}=1.065, p=0.2986\right.$, two-sided paired Student's $t$ test) $(\mathbf{n})$ and test $\left(t_{22}=3.753, p=0.0011\right.$, two-sided paired Student's $t$ test) (o) pathway reveals a time- and pathway-dependent increase in $\gamma$. Note that higher conductance was observed in the test input (o) compared to the control (n) under the "baseline" state, suggesting that the first + second TBS were sufficient to increase $\gamma$. The third TBS triggered a small but discernible further increase in $\gamma$. $\mathbf{q}, \mathbf{r}$ Analysis of the relationships of $\gamma$ with $\operatorname{LTP}\left(p=0.0225, F_{(1,21)}=6.066, F\right.$-test $)(\mathbf{q})$ and decay time of EPSCs $\left(p=0.0712, F_{(1,21)}=3.612, F\right.$-test $)(\mathbf{r})$. Data are presented as mean \pm SEM. Source data are provided as a Source Data file. 


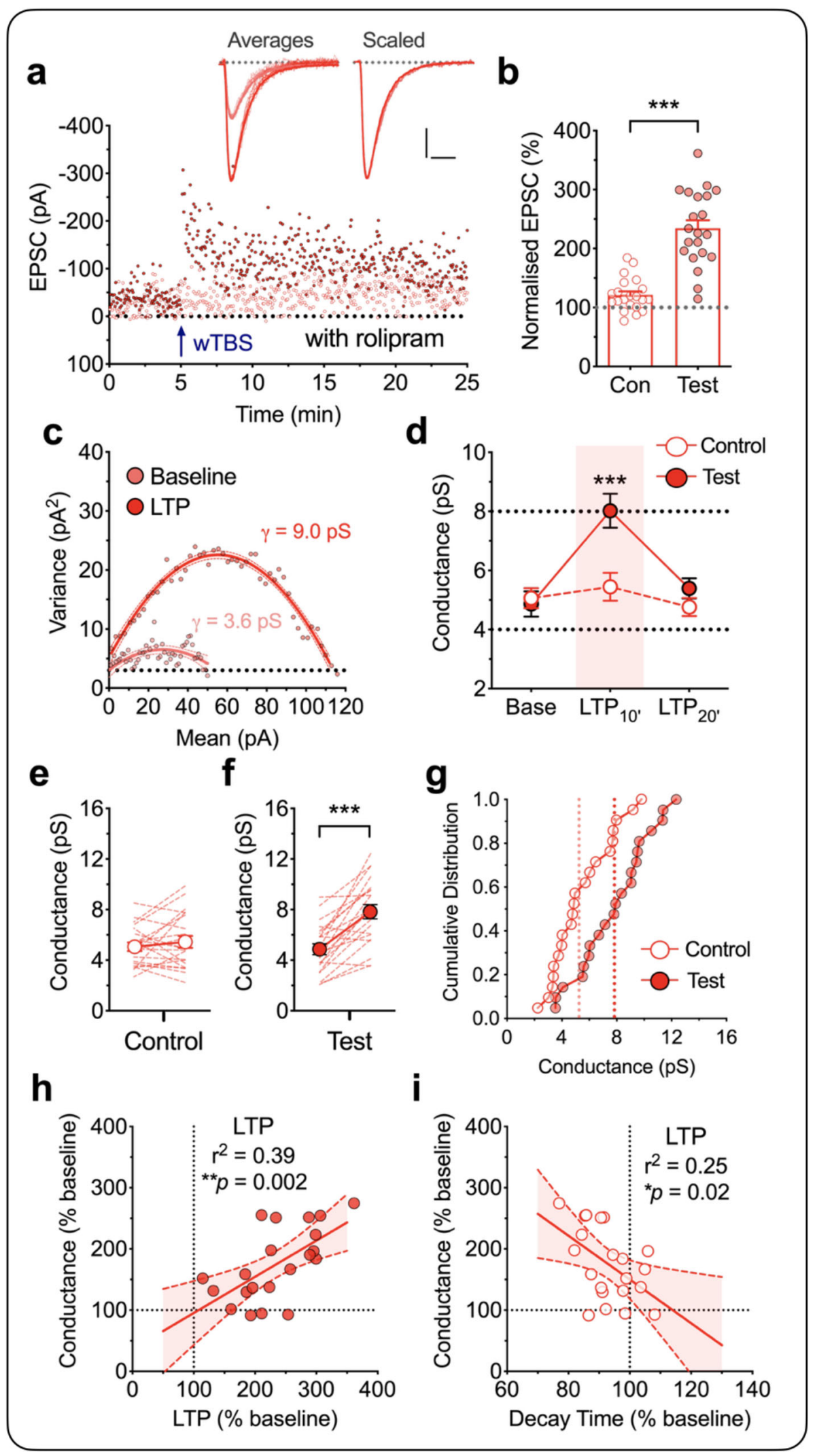

further support the idea that insertion of CP-AMPARs mediates $\operatorname{LTP}_{\gamma}$.

To more specifically test the requirement of PKA for driving alterations in $\gamma$, we included the catalytic subunit of PKA (PKA $\mathrm{C} \alpha 300 \mathrm{U} / \mathrm{mL}$ ) in the patch solution (Fig. 4). This treatment had little effect on the control input that did not receive any wTBS
(Fig. 4a), suggesting that PKA alone has minimal effect on synaptic transmission. However, as was the case with rolipram, the wTBS in the presence of PKA Ca generated a robust potentiation (Fig. 4a) that was associated with an increase in $\gamma$ (Fig. 4b, c). The levels quantified during baseline and 10 min post TBS $\left(\mathrm{LTP}_{10}\right)$ were $5.2 \pm 0.5 \mathrm{pS}$ and $7.8 \pm 0.8 \mathrm{pS}\left(t_{16}=5.80, p<\right.$ 
Fig. 3 Increased AMPA receptor unitary conductance $(\boldsymbol{\gamma})$ during LTP in the presence of rolipram. a-g Equivalent experiments to those illustrated in Fig. 2 for the LTP induced by a wTBS (a single episode of TBS) in the presence of rolipram ( $1 \mu \mathrm{M} ; n=21$ neurons from 15 animals). Scale bars: $10 \mathrm{pA}$ and $10 \mathrm{~ms}$. b Quantification for the levels of LTP for control and test inputs during the $10 \mathrm{~min}$ epoch after the induction (mean $\pm \mathrm{SEM}, t_{20}=7.860, p<0.0001$, twosided paired Student's $t$ test). d-f Statistical analysis between control and test pathways for LTP $_{10}\left(t_{20}=5.901, p<0.0001\right.$, two-sided paired Student's $t$ test) $(\mathbf{d})$ and within pathway analysis for control $\left(t_{20}=0.4416, p=0.6635\right.$, two-sided Student's $t$ test $)(\mathbf{e})$ and test $\left(t_{20}=6.059, p<0.0001\right.$, two-sided paired Student's $t$ test) (f) inputs. $\mathbf{h}$, i Analysis of the relationships of $\gamma$ with LTP ( $p=0.0024, F_{(1,19)}=12.27, F$-test) (h) and decay time of EPSCs ( $p=$ $0.0199, F_{(1,19)}=6.462$, F-test) (i). Data are presented as mean \pm SEM. Source data are provided as a Source Data file.

0.0001 , paired Student's $t$ test; $n=17 / 13$; Fig. 4b). Once again, the increase in $\gamma$ was only transient, since estimates of $\gamma$ made between 10 and $20 \mathrm{~min}$ following the wTBS (i.e. $\mathrm{LTP}_{20^{\circ}}$ ) were not significantly different from baseline $\left(5.3 \pm 0.5 \mathrm{pS} ; t_{16}=0.37, p=\right.$ 0.7163 , paired Student's $t$ test; Fig. $4 \mathrm{~b})$.

To establish whether the increase in $\gamma$ was indeed due to the insertion of CP-AMPARs we used IEM-1460 (IEM, 30 $\mu \mathrm{M}$ ). Previously, we showed that IEM inhibited LTP triggered by a sTBS without affecting LTP triggered by a cTBS ${ }^{26,27}$. Since these two induction protocols activate NMDARs to a similar extent, the effects of IEM is unlikely to be due to a direct action on NMDARs. To establish whether this is indeed the case, we examined the effects of IEM on NMDAR-mediated EPSCs evoked by single pulses and during TBS. IEM had no effect whatsoever on NMDAR-mediated synaptic transmission (Supplementary Fig. 1).

In the presence of bath applied IEM and PKA Ca in the patch pipette, the level of LTP triggered by the wTBS was significantly less than in its absence $(202 \pm 16 \%$ vs. $276 \pm 19 \%$ of baseline, 10 min after wTBS; $t_{31}=3.01, p=0.0052$, unpaired Student's $t$ test; Fig. $4 \mathrm{~d}, \mathrm{~g}$ ), consistent with a component of LTP being generated by the insertion of CP-AMPARs when PKA is activated ${ }^{26,30-32}$. IEM completely prevented the transient increase in $\gamma$ (baseline vs. $\mathrm{LTP}_{10} ; 4.3 \pm 0.5 \mathrm{pS}$ vs. $4.3 \pm 0.5 \mathrm{pS} ; t_{15}=0.08, p=0.9338$, paired Student's $t$ test; Fig. $4 \mathrm{e}, \mathrm{h} ; n=16 / 13$; also see Supplementary Table 1). There was a strong correlation between the increase in $\gamma$ with both the magnitude of LTP (Fig. 4i; $p=0.0021, F_{(1,15)}=$ 13.72 ) and the decrease in $\tau_{\text {decay }}$ (Fig. $4 \mathrm{k} ; p=0.0117, F_{(1,15)}=8.24$ ) when wTBS was delivered in the presence of PKA Ca, but there were no such correlations when IEM was also present (Fig. 4j, l).

In conclusion, we find that activation of PKA, that occurs during (i) a sTBS, (ii) a wTBS in the presence of rolipram or (iii) a wTBS in the presence of the catalytic subunit of PKA, results in the transient insertion of CP-AMPARs and that these receptors are responsible for the increase in $\gamma$ during the initial expression phase of LTP.

The role of CaMKII in $\mathbf{L T P}_{\boldsymbol{\gamma}}$. CaMKII has been demonstrated to be both necessary and sufficient for the induction of LTP ${ }^{10,11}$. Consistent with this notion, when tested using a CaMKII selective antagonist, KN-62 $(10 \mu \mathrm{M})$, we found that both cLTP and sLTP were substantially reduced (Fig. $5 \mathrm{a}, \mathrm{b}$ ). The levels of potentiation of $108 \pm 8 \%$ (after $90 \mathrm{~min}$ of cTBS, $n=4$ slices from individual animals; Fig. 5a) and $105 \pm 7 \%$ (after $120 \mathrm{~min}$ of sTBS, $n=5$ slices; Fig. 5b), respectively, were significantly less than that in the corresponding interleaved untreated control groups that potentiated $155 \pm 5 \%\left(p=0.0010, t_{9}=4.79\right.$; unpaired Student's $t$ test; $n=7$ slices $)$ and $159 \pm 3 \%\left(p=0.0001, t_{13}=6.87\right.$; unpaired Student's $t$ test; $n=10$ slices), but were not significantly different from their respective control inputs $\left(t_{3}=1.50, p=0.2315\right.$ and $t_{4}=0.66 ; p=0.5426$; paired Student's $t$ test).

It has been suggested that the role of CaMKII in LTP involves an increase in $\gamma^{14,15}$. To further examine the role of CaMKII in LTP we interleaved experiments where we applied either active or inactive (heat inactivated) CaMKII $(250 \mathrm{U} / \mathrm{mL})$ via the patch pipette and delivered baseline (low frequency) stimulation to monitor basal synaptic transmission. Consistent with previous reports $^{33,34}$, activated CaMKII, but not inactive CaMKII, was sufficient to potentiate synaptic transmission (Fig. $5 c, d, h$ ). However, this potentiation was not associated with an increase in $\gamma$ (Fig. 5f, g, i) or a change in rise and decay kinetics (Fig. 5e; see also Supplementary Table 1). The respective $\gamma$ values for baseline (i.e. first $5 \mathrm{~min}$ of recording) and $10-15 \mathrm{~min}$ of whole-cell recording were $4.7 \pm 0.6 \mathrm{pS}$ and $4.3 \pm 0.5 \mathrm{pS}\left(t_{14}=0.74, p=\right.$ 0.4740 , paired Student's $t$ test; $n=15 / 12$; Fig. $5 \mathrm{~g}$ ). There was no correlation between $\gamma$ change and either the magnitude of LTP (Fig. 5j; $p=0.2265, F_{(1,13)}=1.61$ ) or $\tau_{\text {decay }}$ (Fig. $5 \mathrm{k} ; p=0.2813$, $\left.F_{(1,13)}=1.26\right)$. We can conclude, therefore, that CaMKII alone can generate substantial potentiation that does not involve any alteration in $\gamma$.

Activation of CaMKII and PKA are both necessary and sufficient for LTP $_{\boldsymbol{\gamma}}$. Since neither PKA alone nor CaMKII alone affected $\gamma$, we explored whether the combination of the two kinases may be sufficient for the effect. We, therefore, patch loaded PKA Ca $(300 \mathrm{U} / \mathrm{mL})$ with either the active or inactive forms of CaMKII $(250 \mathrm{U} / \mathrm{mL})$. In interleaved experiments, we found that PKA $\mathrm{Ca}+$ active CaMKII produced a robust potentiation of synaptic responses, specifically $178 \pm 10 \%$ of baseline when quantified $15 \mathrm{~min}$ after whole-cell (Fig. 6a, b, f). In this case, the effect was also associated with an increase in $\gamma$ (Fig. 6d, e, g). The levels of conductance for the baseline and potentiation (calculated between 10 to $15 \mathrm{~min}$ of recording) were $4.6 \pm 0.4 \mathrm{pS}$ and $6.5 \pm 0.4$ $\mathrm{pS}$, respectively $\left(t_{17}=5.38, p=0.0002\right.$, paired Student's $t$ test; $n=$ 18/15). Again, this effect was only transient, as the $\gamma$ returned to baseline levels within 20-30 min of whole-cell recording (Fig. 6e). In contrast, inactive CaMKII plus PKA Ca, had no significant effect on synaptic transmission (112 $\pm 9 \%$; Fig. $6 \mathrm{~b}, \mathrm{f})$ or on $\gamma(4.2$ $\pm 0.4 \mathrm{pS}$ vs. $4.2 \pm 0.4 \mathrm{pS}, n=16 / 14$; Fig. $6 \mathrm{e}, \mathrm{g}$ ). These results suggest that (i) both CaMKII and PKA are required for and (ii) their combined activity is sufficient for $\mathrm{LTP}_{\gamma}$ at these synapses.

In additional interleaved experiments, the sensitivity to IEM was tested on the potentiation produced by CaMKII plus PKA Ca. Consistent with the involvement of CP-AMPARs, there was a reduced level of potentiation (Fig. $6 \mathrm{~b}, \mathrm{f}$ ) and no change in $\gamma$ in the presence of IEM (Fig. 6e, g). The respective amounts, quantified after $10-15 \mathrm{~min}$ of whole-cell recording, were $128 \pm 9 \%$ of baseline $(p=$ 0.0003 vs. CaMKII + PKA Ca, one-way ANOVA with Bonferroni's correction) and $4.1 \pm 0.4 \mathrm{pS}$ ( $p=0.0009$ vs. CaMKII + PKA Ca, oneway ANOVA with Bonferroni's correction; $n=20 / 15)$.

The correlations between the change in $\gamma$ with the level of LTP and decay times for these experiments are summarized in Fig. $6 \mathrm{~h}-\mathrm{k}$. There was a substantial correlation between the increase in $\gamma$ and the level of potentiation (Fig. 6h) and the decrease in $\tau_{\text {decay }}$ (Fig. 6i) with CaMKII plus PKA Ca but no such correlation was found in the presence of IEM (Fig. 6j, k).

The proportion of synaptically incorporated CP-AMPARs during $\mathbf{L T P}_{\boldsymbol{\gamma}}$. Together, the previous experiments provide multiple lines of evidence that $\mathrm{LTP}_{\gamma}$ is due to the insertion of CP-AMPARs 

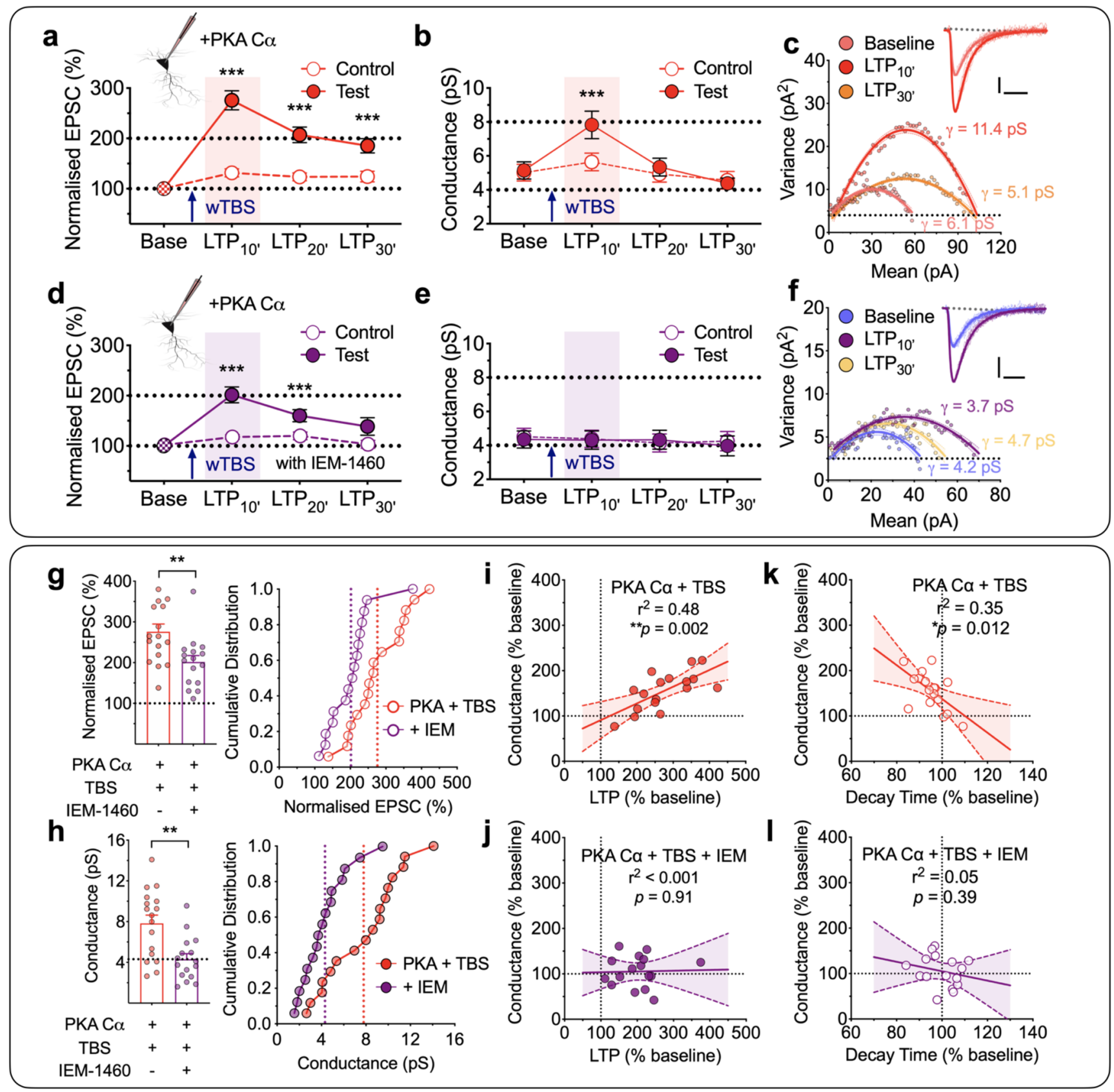

Fig. 4 wTBS with PKA C $\alpha$ transiently increases $\gamma$ via CP-AMPAR insertion. a-b A wTBS in the presence of intracellular PKA C $\alpha$ (300 U/mL) transiently increased $\gamma\left(n=17\right.$ neurons from 13 animals, mean \pm SEM, $F_{(1.931,27.04)}=52.89$ for EPSCs and $F_{(1.863,26.08)}=12.59$ for $\gamma$, one-way repeated measures ANOVA followed by Bonferroni's multiple comparisons test; ${ }^{\star} p<0.05,{ }^{\star \star} p<0.01$ and ${ }^{* \star *} p<0.001$ vs. baseline). EPSCs (a) and $\gamma$ (b) were analyzed in $10-$ min bins. A single episode of TBS (at time marked by an arrow) was delivered to one input (filled symbols) with the second input (open symbols) serving as a control; base = baseline. $\mathbf{c}$ A representative current-variance plot for PKA C $\alpha$ plus wTBS for baseline, the first 10 min $\left(L_{T P} 0^{\prime}\right)$ and the last 10 min of $L T P\left(L T P 30^{\prime}\right)$. Sample traces were obtained from baseline and LTP $10^{\prime}$. Scale bars: $10 \mathrm{pA}$ and $10 \mathrm{~ms}$. d-f Equivalent experiments in the presence of IEM-1460 (IEM, $30 \mu \mathrm{M}$; $n=16$ neurons from 13 animals, $F_{(1.095,13.14)}=25.66$ for EPSCs and $F_{(2.184,26.21)}=0.2547$ for $\gamma$, one-way repeated measures ANOVA followed by Bonferroni's multiple comparisons test; ${ }^{*} p<0.05,{ }^{\star \star} p<0.01$ and ${ }^{\star \star *} p<0.001$ vs. baseline). $\mathbf{g}$, $\mathbf{h}$ Quantification of the levels of LTP $\left(t_{31}=3.006, p=0.0052\right.$, two-sided unpaired Student's $t$ test $)(\mathbf{g})$ and $\left(t_{31}=3.544, p=0.0013\right.$, two-sided unpaired Student's $t$ test $) \gamma(\mathbf{h})$ measured during the 10 min after wTBS with cumulative distributions (right). $n=17$ neurons from 13 animals (PKA C $\alpha+$ wTBS) and 16 neurons from 13 animals (PKA C $\alpha+w T B S+I E M)$ ). i, j Analysis of the relationships between $\gamma$ and LTP for PKA C $\alpha+$ WTBS $\left(p=0.0021, F_{(1,15)}=13.72, F\right.$-test $)(\mathbf{i})$ and PKA C $\alpha+$ wTBS + IEM $\left(p=0.9090, F_{(1,14)}=0.0136, F\right.$-test $)$ (j). k, I Analysis of the relationships between $\gamma$ and EPSC decay time for PKA C $\alpha+$ wTBS $\left(p=0.0117, F_{(1,15)}=8.243, F\right.$-test $)(\mathbf{k})$ and PKA C $\alpha+$ wTBS + IEM $(p$ $=0.3931, F_{(1,14)}=0.7764, F$-test) $(\mathbf{I})$. Data are presented as mean \pm SEM. Source data are provided as a Source Data file.

into synapses that contain CI-AMPARs. In order to determine the relative proportions of each it was necessary to measure $\gamma$ for synapses containing either just CI-AMPARs or just CP-AMPARs, under our recording conditions. To achieve this, we used lentivirusdriven CRISPR/Cas9 expression to delete GluA2 in a fraction of neurons in vivo, allowing a direct comparison between a knock-out (KO) and a wild-type (WT) neuron within each adult brain slice (Fig. 7a). When compared with uninfected neighbouring neurons, the KO cells showed a reduced AMPAR synaptic transmission (Fig. 7b) and an inwardly rectifying current-voltage relationship 

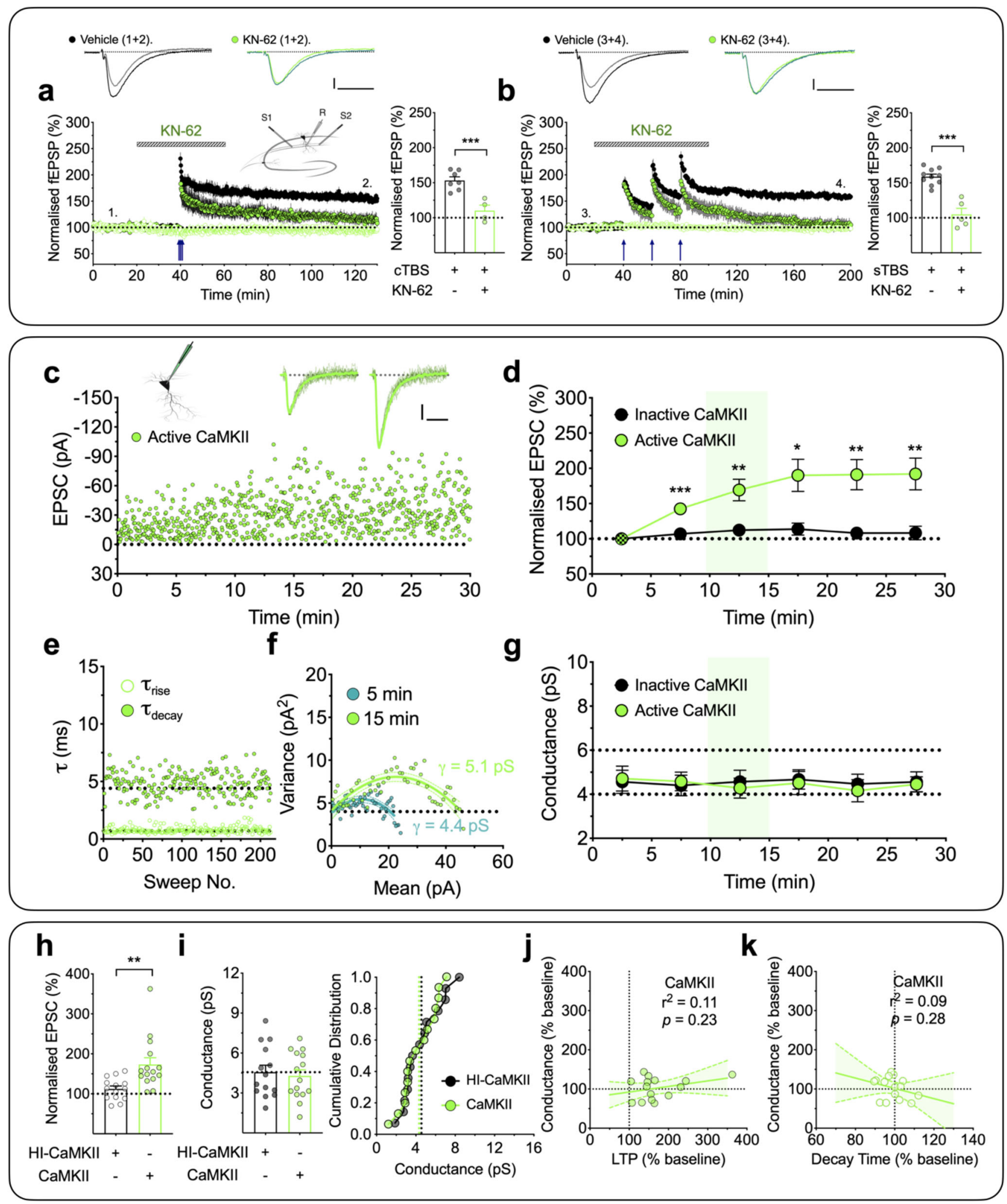

(Fig. 7c, d). The level of $\gamma$ in KO neurons was significantly higher at $17.3 \pm 1.2 \mathrm{pS}(n=16)$ compared to $4.6 \pm 0.4 \mathrm{pS}$ for WT neurons $(n$ $=17 ; t_{31}=10.09, p<0.0001$, unpaired Student's $t$ test, data from 12 animals; Fig. 7e-h). This increase in $\gamma$ in these neurons was associated with a decreased $\tau_{\text {decay }}$ from $6.8 \pm 0.4 \mathrm{~ms}$ in WT to $5.3 \pm 0.4 \mathrm{~ms}$ in KO neurons ( $t_{31}=2.85, p=0.0026$, unpaired Student's $t$ test, Fig. 7i). Assuming that these EPSCs were comprised of 100 and $0 \%$ CPAMPARs, respectively, then the increase in $\gamma$ that we observed during
sLTP can be explained by CP-AMPARs comprising $~ 30 \%$ of the synaptic current during the first $10 \mathrm{~min}$ following LTP induction.

\section{Discussion}

NMDA receptor-dependent LTP has been extensively studied as the primary mechanisms utilized are crucial for the formation of long-term memories. Despite many molecules being discovered 
Fig. 5 CaMKII does not affect $\gamma$. $\mathbf{a}, \mathbf{b}$ CaMKII dependence of both forms of LTP (mean \pm SEM). a cLTP, measured using fEPSP recordings, was inhibited by the CaMKII inhibitor, KN-62 (10 $\mu$ M; $n=4$ animals; green). b sLTP showed a similar sensitivity to KN-62 ( $n=5$ animals). Interleaved control experiments $\left(n=7\right.$ and 10 animals; black) are superimposed. $t_{9}=4.786(p=0.0010 ;$ cTBS $)$ and $t_{13}=6.865,(p<0.0001 ;$ sTBS $)$ by two-sided unpaired Student's $t$ test. The sample traces were obtained at the time indicated by the numbers. Scale bars: $0.2 \mathrm{mV}$ and $10 \mathrm{~ms}$. c A representative whole-cell recording with the inclusion of activated CaMKII $(250 \mathrm{U} / \mathrm{mL})$ in the internal solution. The sample traces are averages of selected records for analysis, superimposed with individual scaled traces (10 successive sweeps, thin lines) after 5 and $15 \mathrm{~min}$ of whole-cell recording. Scale bars: $10 \mathrm{pA}$ and $10 \mathrm{~ms}$. d Pooled results (mean \pm SEM, 5-min bins) for the effects on EPSC (\%) by activated CaMKII ( $n=15$ neurons from 12 animals, $F_{(1.378,19.30)}=10.52, p=0.0021$, one-way repeated measures ANOVA) and interleaved control, heat-inactivated, CaMKII $\left(n=14\right.$ neurons from 11 animals, $F_{(2.585,33.61)}=1.096$, one-way repeated measures ANOVA). Bonferroni's post hoc multiple comparisons test; ${ }^{\star} p<0.05,{ }^{\star \star} p<0.01$ and ${ }^{\star \star \star} p<0.001$ vs. the initial 5 min of whole-cell recording. e Rise times $\left(20-80 \%, \tau_{\text {rise }}\right)$ and decay time constants $\left(\tau_{\text {decay }}\right)$ were plotted for the EPSCs used in the NSFA analysis for the neuron illustrated in $\mathbf{c} . \mathbf{f}$ Current-variance relationships for this neuron used to estimate $\gamma$ over $5 \mathrm{~min}$ epochs starting at 0 and $10 \mathrm{~min}$ after commencing whole-cell recording. $\mathbf{g}$ Time course for the estimates of $\gamma$ for active vs. inactive CaMKII. One-way repeated measures ANOVA; $F_{(3.837,53.72)}=0.2498$ ( $n=15$ neurons from 12 animals, active CaMKII), $F_{(3.706,48.18)}=0.0795$ ( $n=14$ neurons from 11 animals, inactive CaMKII). Bonferroni's post hoc multiple comparisons test; ${ }^{\star} p<0.05,{ }^{\star \star} p<0.01$ and ${ }^{\star \star *} p<0.001$ vs. the initial 5 min of whole-cell recording. $\mathbf{h}$, i Quantification for the levels of LTP $\left(t_{27}=3.125, p=0.0040\right.$, two-sided unpaired Student's $t$ test $)(\mathbf{h})$ and $\gamma\left(t_{27}=0.3539, p=0.7262\right.$, two-sided unpaired Student's $t$ test) (i) measured over a 5 min epoch, commencing 10 min after starting wholecell recording. $\mathbf{j}, \mathbf{k}$ Analysis of the relationships between $\gamma$ and LTP $\left(p=0.2265, F_{(1,13)}=1.611, F\right.$-test $)(\mathbf{j})$ and EPSC decay time $\left(p=0.2813, F_{(1,13)}=1.264, F-\right.$ test) (k) for the active CaMKII experiments. Data are presented as mean \pm SEM. Source data are provided as a Source Data file.

and different aspects of their regulation being uncovered, there are crucial gaps in our knowledge. One relates to the fact that long-term memory requires de novo protein synthesis yet most of our mechanistic understanding of LTP has been obtained from the study of a protein synthesis-independent form of LTP. A second pertains to the fact that much of this understanding has been derived from the study of juvenile animals, where technical issues have permitted more in-depth analysis, whereas most studies of learning and memory are conducted in adult animals. In the present study, we have addressed these issues by studying LTP at CA1 synapses in young adult rodents and have compared induction protocols that are known to activate the protein synthesis-independent (cTBS) and protein synthesis-dependent (sTBS, rolipram + wTBS) forms ${ }^{35,36}$. Using a cTBS protocol, LTP involved the insertion of additional CI-AMPARs, for which activation of CaMKII is both necessary and sufficient. Using a sTBS there was an additional LTP component that involved the transient insertion of CP-AMPARs, for which activation of CaMKII and PKA are both necessary and, in combination, sufficient. The insertion of CP-AMPARs increases AMPA receptor $\gamma$ and this underlies the initial expression of this form of LTP, which we have termed $\mathrm{LTP}_{\gamma}$. The insertion of CP-AMPARs is transient and is replaced by a persistent increase in the number of CI-AMPARs.

Two distinct postsynaptic forms of LTP at CA1 synapses. The division of NMDA receptor-dependent LTP into multiple components was made on the basis of sensitivity to various pharmacological agents and substantiated by genetic studies ${ }^{36}$. In particular, when a single train (tetanus or TBS) is employed, the resultant LTP may be independent of both PKA activation and de novo protein synthesis; this is commonly referred to as LTP1 or E-LTP ${ }^{35,37}$. In contrast, when multiple trains are delivered, with an interval in the order of minutes, then there is often the generation of an additional PKA and de novo protein synthesisdependent component of LTP, which is commonly referred to as LTP2 or L-LTP27,36,38,39. LTP2 is generally assumed to underlie long-term memory formation, that also requires de novo protein synthesis.

NMDA receptor-dependent LTP has also been divided into two distinct postsynaptic mechanisms of expression, one involving an increase in the number of AMPARs without a change in $\gamma$ $\left(\mathrm{LTP}_{\mathrm{N}}\right)$ and the other involving an increase in $\gamma\left(\mathrm{LTP}_{\gamma}\right)^{6}$. Here, one of our goals was to determine whether these separate expression mechanisms specifically relate to LTP1 and LTP2. We found that LTP1 never involved an alteration in $\gamma$ whereas LTP2 invariably did. The increase in $\gamma$ was transient, lasting between 10 and $20 \mathrm{~min}$ and could be fully explained by the insertion of CPAMPARs. In terms of signalling cascades, we found that activation of CaMKII was both necessary and sufficient for LTP1 whereas both CaMKII and PKA were required, and in combination were sufficient, for LTP2 (see model in Fig. 8). Our findings do not conflict with a large body of literature regarding alterations in AMPARs underlying LTP at these synapses and the roles of both CaMKII and PKA (e.g. ${ }^{26,30,40-42}$ ).

The roles of CP-AMPARs and alterations in $\gamma$ in LTP have been controversial $^{6,24,43-45}$. However, these controversies can now be reconciled on the basis of the type of LTP under investigation. Under the conditions of our study, we could readily switch between forms of LTP that do not (LTP1) or do (LTP2) involve a CP-AMPAR component by simply altering the timing between TBS episodes. We saw similar effects when we compared wholecell recordings, using minimal stimulation, with field potential recordings, which provide an average measure of synaptic transmission across a wide range of release probabilities $P(r)$. Therefore, we do not expect that our observations are dependent on the $P(r)$ of the synapse under investigation. However, the extent to which CP-AMPARs are involved in synaptic plasticity is likely to involve additional factors, such as the developmental stage of the animal, the level of stress experienced prior to euthanasia and the precise experimental conditions used, including the stimulus parameters employed $31,32,43,44,46,47$.

We can conclude that LTP1 equates to LTP $_{\mathrm{N}}$ and LTP2 with $\mathrm{LTP}_{\gamma}$. It is important to note, however, that although a compressed induction protocol (cTBS) will ordinarily result in just LTP1/LTP $\mathrm{N}_{\mathrm{N}}$, a spaced protocol will comprise a mixture of LTP1 and LTP2 $\left(\operatorname{LTP}_{\gamma}\right)$, since the initial train will induce LTP1 upon which subsequent trains will add LTP2 under our experimental conditions. The relative proportion of these two components will depend on a variety of conditions, including the interval between the trains, with $\sim 10$ min being optimal for the induction of LTP2 $2^{26}$.

On the mechanism of $\operatorname{LTP}_{\gamma}$. The increase in $\gamma$ is regulated by the c-terminal tail of GluA1 ${ }^{48}$ and could result from a CaMKIIdependent phosphorylation of Ser831 of GluA1 to directly modulate their multiple conductance states ${ }^{14,16}$ and/or by the insertion of CP-AMPARs ${ }^{25}$, since these have a higher single channel conductance than CI-AMPARs ${ }^{17,18}$. Our findings have demonstrated that $\operatorname{LTP}_{\gamma}$ can be explained exclusively by the latter mechanism, since all changes in $\gamma$ were eliminated by IEM. Furthermore, we found that activation of PKA plus CaMKII increased $\gamma$ whereas 

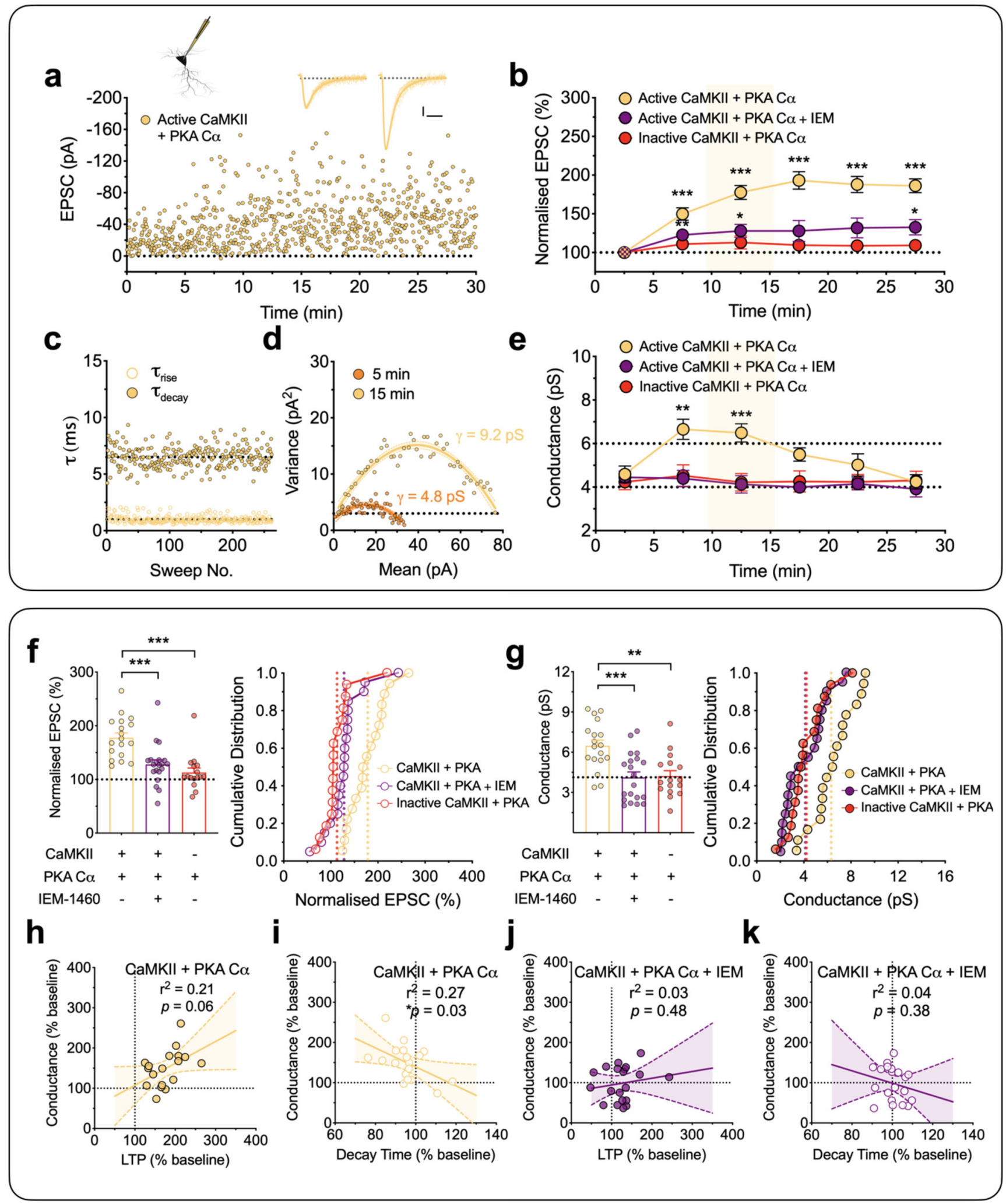

CaMKII alone did not, despite leading to a substantial potentiation. The failure of CaMKII alone to increase $\gamma$, which is contrary to some previous studies ${ }^{14,15}$, could be explained on the basis of the native AMPAR configuration since $\gamma$ alterations are affected by the subunit combination and accessary protein composition of AMPA receptors ${ }^{16,18}$. It is worth noting, however, that whilst activation of CaMKII alone was not sufficient to induce $\mathrm{LTP}_{\gamma}$ its activation was necessary. It is possible, therefore, that phosphorylation of Ser831 of GluA1 is a necessary step for $\operatorname{LTP}_{\gamma}$. Such a mechanism would involve dual phosphorylation of GluA1 on Ser831 and Ser845, which is known to occur ${ }^{49}$. One scenario is that GluA1 is firstly phosphorylated on Ser845 to drive CPAMPARs to peri-synaptic sites, for which considerable evidence already exists ${ }^{30,41,42,49-51}$. From here, they are next 
Fig. 6 CaMKII plus PKA C $\boldsymbol{\alpha}$ results in a transient synaptic insertion of CP-AMPARs and increase in $\boldsymbol{\gamma}$. a-k Equivalent experiments as described in Fig. 5c-k but with the inclusion of activated CaMKII $(250 \mathrm{U} / \mathrm{mL}$ ) plus the catalytic subunit of PKA (PKA C $\alpha, 300 \mathrm{U} / \mathrm{mL})$ in the internal solution. Scale bars: $10 \mathrm{pA}$ and 10 ms. b Pooled results (mean \pm SEM, 5-min bins) for the effects on EPSC (\%) by active CaMKII + PKA C $\alpha\left(n=18\right.$ neurons from 15 animals, $F_{(2.560,43.52)}=38.34$, one-way repeated measures ANOVA), CaMKII + PKA C $\alpha+$ IEM-1460 (IEM, $30 \mu M ; n=20$ neurons from 15 animals, $\left.F_{(2.064,39.22)}=4.079\right)$ and heatinactivated $\mathrm{CaMKII}+\mathrm{PKA} C \alpha\left(n=16\right.$ neurons from 14 animals, $\left.F_{(2.682,40.23)}=1.301\right)$. Bonferroni's post hoc multiple comparisons test; ${ }^{\star} p<0.05,{ }^{\star \star} p<0.01$ and ${ }^{* \star *} p<0.001$ vs. the initial 5 min of whole-cell recording. e Time course for the estimates of $\gamma$. One-way repeated measures ANOVA followed by Bonferroni's multiple comparisons test $\left({ }^{\star} p<0.05,{ }^{*} p<0.01\right.$ and ${ }^{* \star *} p<0.001$ vs. the initial 5 min of whole-cell recording); $F_{(3.219,54.72)}=9.927(\mathrm{CaMKII}+\mathrm{PKA} C \alpha)$, $F_{(4.067,77.28)}=0.5990\left(\right.$ CaMKII + PKA C $\alpha+$ IEM) and $F_{(3.587,53.80)}=0.1366$ (heat-inactivated CaMKII + PKA C $\left.\alpha\right)$. Quantification for the levels of LTP $\left(F_{(2,51)}=\right.$ 14.19) (f) and $\gamma\left(F_{(2,51)}=9.210\right)(\mathbf{g})$. One-way ANOVA followed by Bonferroni's multiple comparisons test; ${ }^{*} p<0.05$, ${ }^{* *} p<0.01$ and ${ }^{* \star *} p<0.001$. $\mathbf{h}, \mathbf{i}$ Analysis of the relationships between $\gamma$ and $\operatorname{LTP}\left(p=0.0618, F_{(1,16)}=4.073, F\right.$-test $)(\mathbf{h})$ and EPSC decay time $\left(p=0.0340, F_{(1,16)}=5.440, F\right.$-test $)(\mathbf{i})$ for CaMKII + PKA $\mathrm{C} \alpha . \mathbf{j}, \mathbf{k}$ Analysis of the relationships between $\gamma$ and LTP $\left(p=0.4775, F_{(1,18)}=0.5263, F\right.$-test $)(\mathbf{j})$ and EPSC decay time $\left(p=0.3760, F_{(1,18)}=0.8241, F\right.$-test $)(\mathbf{k})$ for CaMKII + PKA C $\alpha+$ IEM. Data are presented as mean \pm SEM. Source data are provided as a Source Data file.

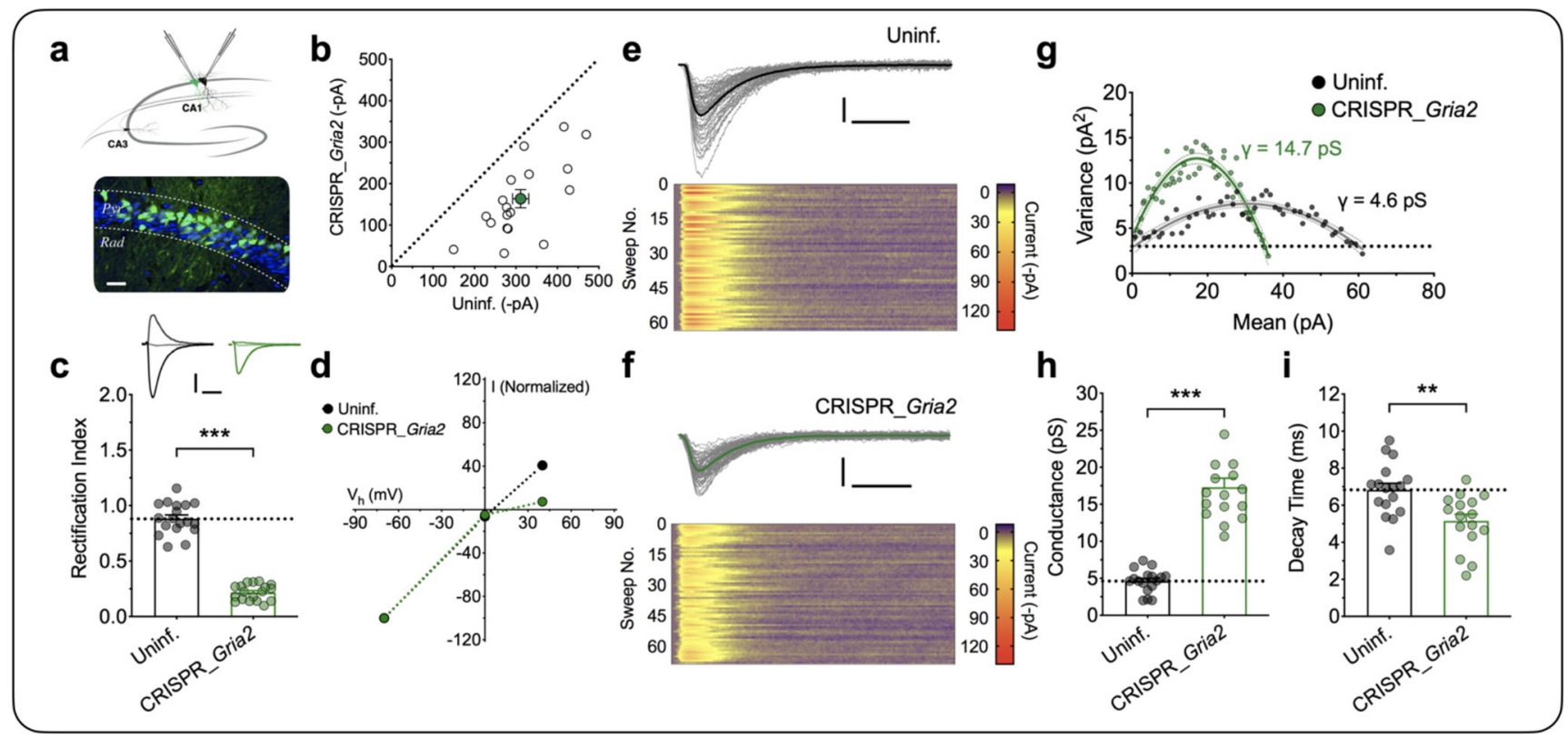

Fig. 7 CP-AMPAR characterization in CRISPR_Gria2 knock-out neurons. a Schematic of dual whole-cell recordings for the CRISPR_Gria2 knock-out and neighbouring uninfected (Uninf.) neurons. Sparse expression following stereotactic lentivirus injection detected by co-expressed EGFP (green); blue, DAPI staining; Pyr, stratum pyramidale; Rad, stratum radiatum. Scale bar $=30$ microns. b Scatterplot shows amplitudes of AMPAR EPSCs for each pair recorded simultaneously (open circles) and the mean \pm SEM (filled circle; $n=18$ pairs from 12 animals). c, d Quantification of the rectification index for pharmacologically isolated AMPAR-mediated EPSCs and the corresponding current-voltage relationship ( $m e a n \pm S E M, n=18$ pairs from 12 animals, $t_{17}=$ 17.38, $p<0.0001$, two-sided paired Student's $t$ test). Scale bars: $100 \mathrm{pA}$ and $10 \mathrm{~ms}$. e-g Representative traces to measure the $\gamma$ for the control and CRISPR_Gria2 knock-out neurons. Individual traces (thin lines) superimposed with the average. Scale bars: 30 pA and 10 ms. The lower panels are corresponding colour-coded images of all sweeps used in the NSFA $(\mathbf{g})$. h, i Quantification of $\gamma\left(t_{31}=10.09, p<0.0001\right.$, two-sided unpaired Student's $t$ test $)$ and decay time ( $t_{31}=3.273, p=0.0026$, two-sided unpaired Student's $t$ test) constants ( $n=17$ and 16 for Uninf. vs. CRISPR_Gria2 knock-out neurons from 12 animals). Data are presented as mean \pm SEM. Source data are provided as a Source Data file.

phosphorylated on Ser831 to drive them into the synapse. In this model, both phosphorylation steps are required for synaptic $\gamma$ to increase because they are regulating different trafficking steps on route to the synapse. In which case, CaMKII should not be able to increase $\gamma$ further in neurons lacking GluA2 because CP-AMPARs are already synaptically expressed. Future work could address this and other aspects of the temporal sequence and consequences of PKA and CaMKII-dependent phosphorylation of GluA1 for LTP.

Our data are compatible with an exchange of a subset of CIAMPARs for CP-AMPARs. The latter could be explained by a mechanism involving the $\mathrm{Ca}^{2+}$ sensor PICK1 $1^{52,53}$, which has been shown to bind and internalize GluA2-containing AMPARs to enable the insertion of CP-AMPARs during LTP ${ }^{54}$. The next step involves the replacement of the newly inserted CPAMPARs with CI-AMPARs, a process that requires baseline (low frequency) synaptic activation ${ }^{26,43}$ and probably involves $\mathrm{Ca}^{2+}$ permeation through the CP-AMPARs themselves ${ }^{55}$. The rapid replacement of CP-AMPARs with CI-AMPARs was originally described at excitatory synapses onto cerebellar stellate neurons from P18-P20 rats ${ }^{56}$. At this synapse, high-frequency stimulation (tetanus) induces CP-AMPARs to be replaced with the equivalent number of $\mathrm{CI}$ forms resulting in a reduction in the synaptic current by a third, reflecting lower $\gamma$ of the latter form. We observed an initial reduction in EPSC amplitude following the triggering of LTP, which might be explained, in part, by a one-toone exchange of CP-AMPARs for CI-AMPARs. Additionally, the transient expression of CP-AMPARs could trigger an increase in the number of AMPAR slots at synapses that enables an increase in the number of CI-AMPARs above and beyond what can occur during LTP1.

Since CP-AMPARs increase synaptic conductance why does there need to be an exchange for a greater number of CI- 


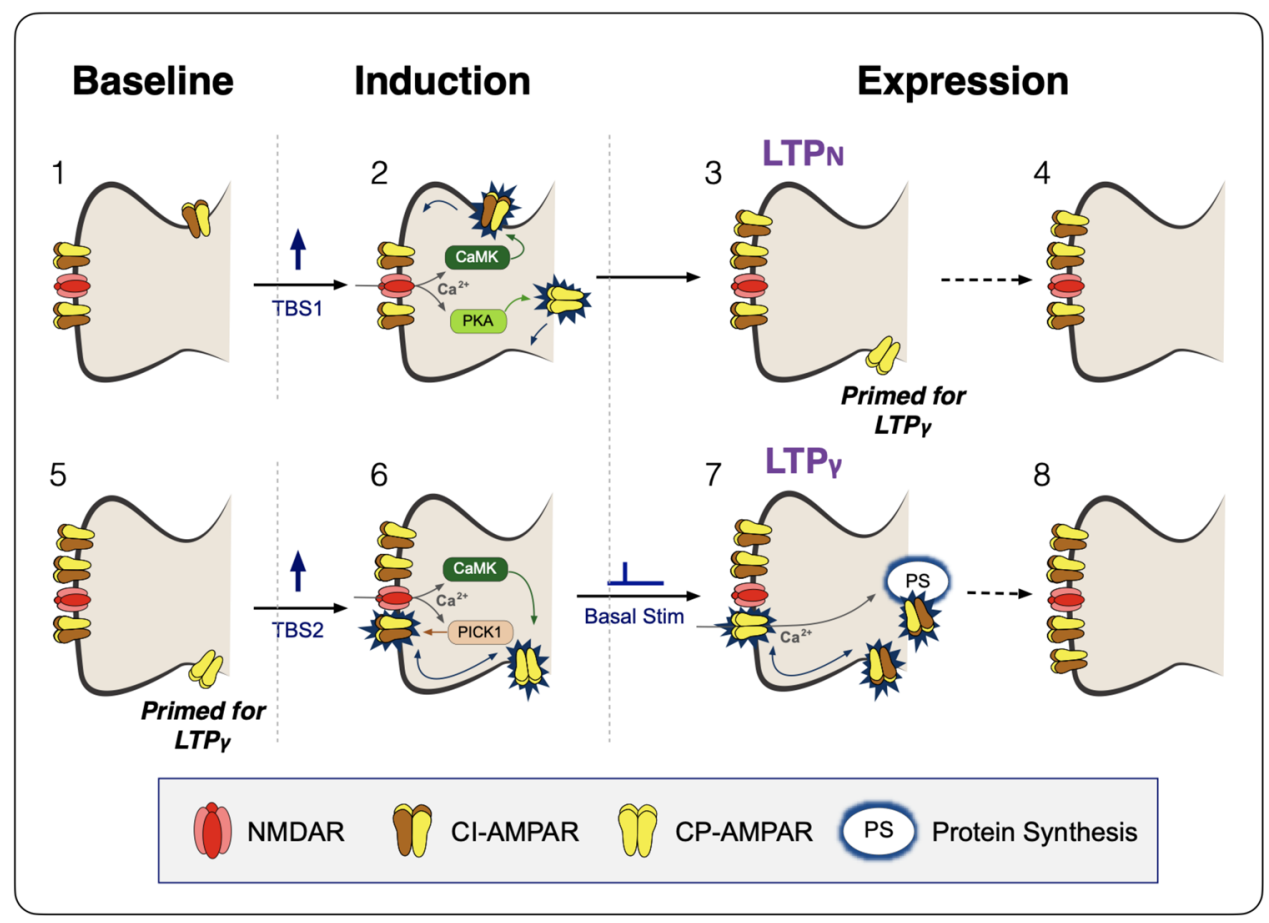

Fig. 8 Schematic outlining the induction of two mechanistically distinct forms of LTP. 1 Under baseline conditions synaptic transmission is mediated by GluA2-containing, calcium-impermeable (CI)-AMPARs, two shown for simplicity. $\mathbf{2}$ The first theta-burst stimulation (TBS) activates NMDA receptors (NMDARs) and this drives more Cl-AMPARs into the synapse by lateral diffusion from a peri-synaptic pool, via a process that involves CaMKII. PKA is also activated (via adenyl cyclase, not shown) and this induces the process of inserting GluA2-lacking calcium-permeable (CP)-AMPARs into peri-synaptic sites on the plasma membrane. 3 LTP is expressed by the increase in number of Cl-AMPARs (LTP ${ }_{N}$ ) but synapses also become primed for LTP $\gamma$ by the availability of peri-synaptic CP-AMPARs. 4 Within $\sim$ h, the peri-synaptic CP-AMPARs are removed and, presumably, degraded. 6 If a second TBS is delivered whilst the synapses are still primed (5) then NMDAR activation drives the peri-synaptically located CP-AMPARs into the synapse, via a CaMKIIdependent process. This might involve an exchange of CP-AMPARs for Cl-AMPARs, which are removed from the synapse via a mechanism triggered by PICK1. 7 These CP-AMPARs increase synaptic strength due to their higher single channel conductance (LTP $)$. However, their dwell time in the synapses is quite short $(\sim 15 \mathrm{~min})$ before they are removed. If synapses remain active, such as by basal stimulation, activation of the transiently available, synaptic CP-AMPARs triggers protein synthesis and the insertion of more Cl-AMPARs (8), which can extend the expression of LTP for long periods.

AMPARs to maintain the enhanced synaptic response? One possibility is that the expression of CP-AMPARs at these synapses needs to be restricted in time due to potential excitotoxicity ${ }^{57}$. Therefore, they can only provide a transient mechanism of expression whilst triggering the more persistent switch resulting in a larger number of CI-AMPARs.

Developmental regulation of the expression mechanisms of LTP. There is strong evidence that the expression mechanisms of LTP are developmentally regulated. The co-existence of two mechanisms involving the insertion of CI-AMPARs and CPAMPARs can account for the LTP at P14 $4^{6}$ and in young adults, as observed herein. However, at around P7, LTP is associated with a decrease in $\gamma^{22}$, which is most likely explained by the replacement of CP-AMPARs with a larger number of CI-AMPARs. Early in development there is also the initial insertion of CP-AMPARs into synapses ${ }^{55}$ that appear to lack AMPARs altogether; so-called "silent" synapses ${ }^{58,59}$. A potential scenario is as follows: first synapses acquire CP-AMPARs, next these are replaced by more CI-AMPARs. Thereafter LTP can increase the number of these CI-AMPARs via two mechanisms, one of which involves the transient insertion of CP-AMPARs and one that does not.

There have been far fewer studies regarding the mechanisms of synaptic plasticity in tissues obtained from adult animals compared to juvenile animals, although most learning and memory studies are conducted in adult animals. This is a concern when attempting to relate mechanisms of synaptic plasticity to learning and memory. Our present study, conducted exclusively in tissue from young adult animals, shows that two distinct forms of synaptic plasticity can be readily induced simply by altering the patterns of activation. Our result that a cTBS protocol induces LTP that does not involve an alteration in $\gamma$ is consistent with another study in adult animals ${ }^{24}$. Our finding that a sTBS induces an additional component of LTP that involves an increase in $\gamma$ is the first evidence that such a process occurs beyond early developmental stages.

Functional significance of two forms of LTP. This raises the question as to why there are two distinct mechanisms to increase the synaptic complement of CI-AMPARs. Previous work has shown that the insertion of CP-AMPARs is specifically associated with the PKA and protein synthesis component of $\mathrm{LTP}^{27}$. It is reasonable to assume, therefore, that the transient insertion of CP-AMPARs is part of the machinery that triggers de novo protein synthesis and the consequential morphological changes (spine enlargement and/or new spine formation). In contrast, in the absence of de novo protein synthesis, the increase in synaptic CI-AMPAR number can support increased synaptic efficacy. Although both processes can increase synaptic strength lasting many hours in vitro, it seems probably that only the protein synthesis-dependent form triggers synaptic changes that underpin long-lasting memories (lasting from days to lifetimes). Indeed, it has been shown that spaced training with access to reward enhances the persistence of memory, and treatment with rolipram after training enhances memory retention ${ }^{60}$. It seems likely that PKA triggers protein synthesis by phosphorylating 
GluA1 on S845 to promote the insertion of CP-AMPARs and by phosphorylating other regulatory targets and that together these regulate gene expression. The requirement for PKA to trigger the protein synthesis-dependent form of LTP also provides the opportunity for extensive neuromodulation. Neurotransmitters, such as noradrenaline and dopamine, and stress hormones, such as corticosterone, may, via the insertion of CP-AMPARs, augment protein synthesis-dependent LTP to enhance and/or prolong the persistence of the associated memory (e.g., 30,31,42,60-62).

Concluding remarks. We have identified the molecular basis of two independent forms of LTP that co-exist at hippocampal synapses in young adult animals, the occurrence of which is controlled by the patterns of synaptic activation during induction. The existence of these two distinct LTP mechanisms goes a long way in explaining many of the controversies that have plagued the field. LTP1 can be induced by a cTBS and involves the insertion of CI-AMPARs, and for this to occur activation of CaMKII is both necessary and sufficient. A sTBS, however, triggers both LTP1 and LTP2. This latter form of LTP involves the transient insertion of CP-AMPARs and this requires activation of PKA in addition to CaMKII.

\begin{abstract}
Methods
Hippocampal slice preparation. Transverse hippocampal slices $(400 \mu \mathrm{m})$ were prepared from male Sprague-Dawley rats (1-3 months of age). Animals were anesthetized with isoflurane and euthanised by decapitation in accordance with UK Animals (Scientific Procedures) Act of 1986. The brain was then removed and placed in ice-chilled slicing solution that contained $(\mathrm{mM}): 124 \mathrm{NaCl}, 3 \mathrm{KCl}, 26$ $\mathrm{NaHCO}_{3}, 1.25 \mathrm{NaH}_{2} \mathrm{PO}_{4}, 10 \mathrm{MgSO}_{4}, 10 \mathrm{D}$-glucose and $1 \mathrm{CaCl}_{2}$, saturated with $95 \% \mathrm{O}_{2}$ and $5 \% \mathrm{CO}_{2}$. The hippocampi were rapidly isolated from the brain and sliced using a vibratome (Microslicer) while maintained in the slicing solution. The CA3 region was removed to suppress the upstream neuronal excitability, and the slices were transferred to an incubation chamber that contained the recording solution (artificial cerebrospinal fluid, ACSF; mM): $124 \mathrm{NaCl}, 3 \mathrm{KCl}, 26 \mathrm{NaHCO}_{3}$ $1.25 \mathrm{NaH}_{2} \mathrm{PO}_{4}, 2 \mathrm{MgSO}_{4}, 10 \mathrm{D}$-glucose and $2 \mathrm{CaCl}_{2}$ (carbonated with $95 \% \mathrm{O}_{2}$ and $5 \% \mathrm{CO}_{2}$ ). Slices were allowed to recover at $32-34^{\circ} \mathrm{C}$ for $30 \mathrm{~min}$, and then maintained at $26-28^{\circ} \mathrm{C}$ for a minimum of $1 \mathrm{~h}$ before recordings were made.
\end{abstract}

Field excitatory postsynaptic potential (fEPSP) recordings. The extracellular electrophysiology was performed in both interface and submerged type chambers maintained at $32^{\circ} \mathrm{C}$, and continuously perfused at $2-4 \mathrm{~mL} / \mathrm{min}$ with oxygenated ACSF. The slope of evoked fEPSPs $(V / s)$ was measured in the CA1 region of hippocampal slices and bipolar stimulating electrodes were used at a constant voltage intensity $(0.1 \mathrm{~ms}$ pulse width) throughout the experiments. Signals were amplified using Axopatch 1D (Molecular Devices) and digitized with BNC-2110 (National Instruments) A/D board at a sampling rate of $20 \mathrm{kHz}$. Recordings were monitored and analyzed using WinLTP v2.363. Each specific experiment was conducted on a single slice from an animal, so the $n$-value reflects both the number of slices and animals used.

Two independent Schaffer collateral-commissural pathways (SCCPs) were stimulated alternately to obtain the evoked synaptic responses, each at a constant baseline frequency of between 0.033 and $0.1 \mathrm{~Hz}$. Following a stable baseline period of at least $20 \mathrm{~min}$, LTP was induced using theta-burst stimulation (TBS) delivered at the same basal stimulus intensity. An episode of TBS comprises 5 bursts at $5 \mathrm{~Hz}$, with each burst composed of 5 pulses at $100 \mathrm{~Hz}$. For LTP induced by compressed TBS (cTBS), three TBS episodes were delivered with an inter-episode interval (IEI) of $10 \mathrm{~s}$. For spaced TBS (sTBS), the same number of episodes were given with an IEI of 10 min (see Fig. 1b). Representative sample traces are an average of 5 consecutive responses, collected from typical experiments (stimulus artefacts were blanked for clarity)

Whole-cell patch clamp recording. Whole-cell recording was made with ACSF that contained $50 \mu \mathrm{M}$ picrotoxin (Abcam) and $20 \mu \mathrm{M}(+)$-bicuculline (Hello Bio) to prevent $\mathrm{GABA}_{\mathrm{A}}$ receptor mediated contribution. CAl pyramidal cells were visualized with IR-DIC optics (Zeiss). The whole-cell solution comprised (mM): $8 \mathrm{NaCl}, 130 \mathrm{CsMeSO}_{3}, 10$ HEPES, 0.5 EGTA, $4 \mathrm{Mg}$-ATP, $0.3 \mathrm{Na}_{3}$-GTP, 5 QX-314 and 0.1 spermine. The $\mathrm{pH}$ was adjusted to $7.2-7.3$ with $\mathrm{CsOH}$ and osmolarity was set to 285-290 mOsm/L. The peak amplitude of evoked EPSCs (pA) was monitored and analyzed using WinLTP v2.363. Two independent SCCPs were stimulated alternately, each at a baseline frequency of $0.1-0.5 \mathrm{~Hz}$. Borosilicate glass pipettes were fire-polished with a final resistance of $2-4 \mathrm{M} \Omega$. Access resistance $\left(R_{\mathrm{A}}\right)$ was estimated by fitting whole-cell capacitance current with a double exponential, and experiments were only accepted for analysis if $R_{\mathrm{A}}$ varied by $<15 \%$. $R_{\mathrm{A}}$ values were
$8.8 \pm 0.3 \mathrm{M} \Omega$; range from 6.2 to $12.8 \mathrm{M} \Omega$. Signals were amplified using an Axopatch $200 \mathrm{~B}$ (Molecular Devices), filtered at $2-5 \mathrm{kHz}$, and digitized at $20 \mathrm{kHz}$ using a BNC-2110 (National Instruments) A/D board.

Cells were voltage-clamped at $-70 \mathrm{mV}$ throughout unless otherwise indicated. LTP was induced using TBS delivered at basal stimulus intensity while in currentclamp mode, and was triggered within $10 \mathrm{~min}$ of whole-cell to prevent the dialysis effect. In some experiments, the PKA catalytic subunit (PKA Ca, $300 \mathrm{U} / \mathrm{mL}$ ) and/ or CaMKII $(250 \mathrm{U} / \mathrm{mL})$ were included in the internal solution. CaMKII was activated ( $1 \times$ NEBuffer for Protein Kinases; $50 \mathrm{mM}$ Tris-HCl, $10 \mathrm{mM} \mathrm{MgCl}_{2}$, $0.1 \mathrm{mM}$ EDTA, $2 \mathrm{mM}$ DTT and $0.01 \%$ Brij 35; $200 \mu \mathrm{M}$ ATP, $1.2 \mu \mathrm{M}$ calmodulin and $2 \mathrm{mM} \mathrm{CaCl}$; incubated for $10 \mathrm{~min}$ at $\left.30^{\circ} \mathrm{C}\right)$ or heat-inactivated $\left(65^{\circ} \mathrm{C}\right.$ for 20 min) as described in the suppliers' manual (New England Biolabs). It is a $\mathrm{Ca}^{2}$

+/calmodulin-dependent, truncated monomer (1-325 amino acid residues) of the a subunit, isolated from Spodoptera frugiperda (Sf9) cells infected with recombinant baculovirus carrying the truncated rat CaMKII (New England Biolabs).

To ensure recording stability, extracellular field EPSPs were simultaneously monitored as described previously ${ }^{26}$. Peak amplitude $(\mathrm{pA})$ and initial slope $(\mathrm{V} / \mathrm{s})$ of EPSCs and fEPSPs were measured, and displayed on-line, using WinLTP v2.363. Whole-cell recordings were initiated following collection of at least $10 \mathrm{~min}$ of stable baseline assessed by extracellular recordings.

Peak-scaled, non-stationary fluctuation analysis (NSFA). The unitary conductance $(\gamma)$ of AMPA receptors was estimated using NSFA according to ref. ${ }^{6}$ (see also ${ }^{19-21}$ ). Whole-cell responses were carefully selected for analysis using WinWCP v5.1 (University of Strathclyde, Glasgow) and Mini Analysis v6.0 (Synaptosoft) software on the basis of the following criteria: first, precise alignment of traces on the rise phase; second, no contamination by spontaneous or polysynaptic currents; third, complete decay from the peak EPSCs. The traces were analyzed and the variance of the decay was plotted as a function of the amplitude at that time point. The $x$-axis was divided into 50-bins of equal current decrement from the peak. The single channel conductance was estimated by fitting the plot to a second polynomial equation, $\sigma^{2}=i I-I^{2} N+b_{1}$, where $\sigma^{2}$ is the variance, $I$ is the mean current $N$ is the number of channels activated, $i$ is the single channel current and $b_{1}$ is the background noise. In the conductance conversion (i.e. $\gamma=i / V$ ), the driving force $(V)$ is the difference between the holding $(-70 \mathrm{mV})$ and reversal potential (assumed to be $0 \mathrm{mV}$ ).

The kinetics of the mean EPSC from each neuron was estimated in Clampfit v10.1 (Molecular Devices) by measuring $20-80 \%$ rise time $\left(\tau_{\text {rise }}\right)$ and the time constant for the decay $\left(\tau_{\text {decay }}\right)$. Representative sample traces are the averages of all of the traces that were selected for analysis, superimposed with individual peakscaled traces (10 successive sweeps), unless otherwise stated. Stimulus intensity was set to obtain a sporadic observation of transmission failures but high enough to obtain a reliable estimate of $\gamma$.

Plasmid constructs and lentivirus production. The following oligonucleotide sequences were used to generate single guide RNA (sgRNA) for GluA2 knockout: forward $\left(5^{\prime}\right.$ to $\left.3^{\prime}\right)$ CACC G ctaacagcatacagataggt; reverse $\left(5^{\prime}\right.$ to $\left.3^{\prime}\right)$ AAAC acctatctgtatgctgttag $\mathrm{C}^{64}$. These were annealed and ligated into the lentivirus backbone developed by the Zhang lab ${ }^{65}$. The construct was modified and used with the CaMKIIa promoter for Cas9-P2A-EGFP expression.

Lentivirus was produced by transfecting Lenti-X $293 \mathrm{~T}$ cells (Takara Bio) with pMD2.G, psPAX2 and lentiCRISPR ${ }^{65}$. The 293 T cells were maintained in serumfree UltraCULTURE media (supplemented with $4 \mathrm{mM}$ L-glutamine, $2 \mathrm{mM}$ GlutaMAX-I, $0.1 \mathrm{mM}$ MEM non-essential amino acids, $1 \mathrm{mM}$ sodium pyruvate, $1 \times$ penicillin/streptomycin). Three days after transfection, the supernatant was filter sterilized $(0.45 \mu \mathrm{m}$ pore membrane, Millipore) and ultracentrifuged at $110,000 \times g$ (Beckman Coulter) with an additional sucrose filtration. The lentivirus pellet was resuspended in Dulbecco's PBS and kept at $-80^{\circ} \mathrm{C}$.

In vivo stereotactic injections and dual whole-cell recordings. The surgical procedure was performed under sterile conditions in accordance with the Institutional Animal Care and Use Committee of Seoul National University. Male C57BL/6 mice (2-3 months of age) were anesthetized by intraperitoneal injection of a ketamine $(130 \mathrm{mg} / \mathrm{kg}$ body weight $)$ and xylazine $(10 \mathrm{mg} / \mathrm{kg})$ mixture. The anesthetized mice were immobilized on a stereotactic apparatus and the lentivira medium $\left(0.5 \mu \mathrm{L}\right.$ per each at a flow rate of $\left.0.1 \mu \mathrm{L} / \mathrm{min} ; 5 \times 10^{9} \mathrm{TU} / \mathrm{ml}\right)$ was bilaterally injected at CA1 area using a microinjection syringe (Hamilton). The coordinates used were $-1.7 \mathrm{~mm}$ posterior, $\pm 1.2 \mathrm{~mm}$ lateral to bregma and $-1.5 \mathrm{~mm}$ below the skull surface.

Following 4-6 weeks of expression, the hippocampal slices were prepared and whole-cell recordings were made as described above. EGFP-positive and neighbouring uninfected neurons were identified by epifluorescence microscopy and compared by dual whole-cell recordings. Rectification index was measured as described in ref. ${ }^{26}$. AMPAR currents were isolated using a mixture of D-AP5 (100 $\mu \mathrm{M})$ and L-689,560 $(5 \mu \mathrm{M})$. The index was calculated by taking the responses from $-70,0$ and $+40 \mathrm{mV}$ of holding voltages. Following the recordings, brain slices were PFA-fixed, stained with DAPI, and imaged on a confocal microscope (Leica SP8). 
Compounds. Drugs were prepared as frozen stock solutions (stored below $-20^{\circ} \mathrm{C}$ ). Compounds were as follows: $N, N, H$,-Trimethyl-5-[(tricyclo[3.3.1.13,7] dec-1ylmethyl)amino]-1-pentanaminium bromide hydrobromide (IEM-1460; Hello Bio); 4-(3-(cyclopentyloxy)-4-methoxyphenyl)pyrrolidin-2-one (rolipram; Abcam); 4-[(2 S)-2-[(5-isoquinolinylsulfonyl)methylamino]-3-oxo-3-(4-phenyl-1piperazinyl)propyl] phenyl isoquinolinesulfonic acid ester (KN-62; Tocris and Hello Bio); D-AP5 (Hello Bio); L-689,560 (Tocris); a catalytic subunit of protein kinase A (PKA Ca, New England Biolabs); $\mathrm{Ca}^{2+} /$ calmodulin-dependent protein kinase II (CaMKII, New England Biolabs).

Statistical analysis. All treatment groups were interleaved with control experiments. Data are presented as mean \pm SEM (standard error of the mean). Responses were normalized to the baseline prior to LTP induction unless otherwise stated. Statistical significance was assessed using (two-tailed) paired or unpaired Student's $t$ tests or one-way ANOVA as appropriate using Graphpad Prism 8. Adjustments were made for multiple comparisons using Bonferroni's correction. The level of significance is denoted on the figures as follows: ${ }^{*} p<0.05,{ }^{*} p<0.01$ and ${ }^{* * *} p<$ 0.001 .

Reporting summary. Further information on research design is available in the Nature Research Reporting Summary linked to this article.

\section{Data availability}

All original data are available upon reasonable request from the authors; the values for data underlying the Figures are provided as a Source Data file. Source data are provided with this paper.

Received: 2 July 2020; Accepted: 8 December 2020;

Published online: 18 January 2021

\section{References}

1. Bliss, T. V. P. \& Collingridge, G. L. A synaptic model of memory: long-term potentiation in the hippocampus. Nature 361, 31-39 (1993).

2. Choi, J.-H. et al. Interregional synaptic maps among engram cells underlie memory formation. Science 360, 430-435 (2018).

3. Josselyn, S. A. \& Tonegawa, S. Memory engrams: recalling the past and imagining the future. Science 367, 39 (2020).

4. Collingridge, G. L., Kehl, S. J. \& McLennan, H. Excitatory amino acids in synaptic transmission in the Schaffer collateral-commissural pathway of the rat hippocampus. J. Physiol. 334, 33-46 (1983).

5. Davies, S. N., Lester, R. A., Reymann, K. G. \& Collingridge, G. L. Temporally distinct pre- and post-synaptic mechanisms maintain long-term potentiation. Nature 338, 500-503 (1989).

6. Benke, T. A., Lüthi, A., Isaac, J. T. \& Collingridge, G. L. Modulation of AMPA receptor unitary conductance by synaptic activity. Nature 393, 793-797 (1998).

7. Shi, S. H. et al. Rapid spine delivery and redistribution of AMPA receptors after synaptic NMDA receptor activation. Science 284, 1811-1816 (1999).

8. Collingridge, G. L., Isaac, J. T. R. \& Wang, Y. T. Receptor trafficking and synaptic plasticity. Nat. Rev. Neurosci. 5, 952-962 (2004).

9. Huganir, R. L. \& Nicoll, R. A. AMPARs and synaptic plasticity: the last 25 years. Neuron 80, 704-717 (2013).

10. Lisman, J., Yasuda, R. \& Raghavachari, S. Mechanisms of CaMKII action in long-term potentiation. Nat. Rev. Neurosci. 13, 169-182 (2012).

11. Herring, B. E. \& Nicoll, R. A. Long-term potentiation: from CaMKII to AMPA receptor trafficking. Annu. Rev. Physiol. 78, 351-365 (2016).

12. Lledo, P. M., Zhang, X., Südhof, T. C., Malenka, R. C. \& Nicoll, R. A. Postsynaptic membrane fusion and long-term potentiation. Science 279, 399-403 (1998).

13. Borgdorff, A. J. \& Choquet, D. Regulation of AMPA receptor lateral movements. Nature 417, 649-653 (2002).

14. Derkach, V., Barria, A. \& Soderling, T. R. Ca2+/calmodulin-kinase II enhances channel conductance of alpha-amino-3-hydroxy-5-methyl-4isoxazolepropionate type glutamate receptors. Proc. Natl Acad. Sci. USA 96, 3269-3274 (1999).

15. Poncer, J.-C., Esteban, J. A. \& Malinow, R. Multiple mechanisms for the potentiation of AMPA receptor-mediated transmission by alpha-Ca2 +/calmodulin-dependent protein kinase II. J. Neurosci. 22, 4406-4411 (2002).

16. Kristensen, A. S. et al. Mechanism of Ca2+/calmodulin-dependent kinase II regulation of AMPA receptor gating. Nat. Neurosci. 14, 727-735 (2011).

17. Verdoorn, T. A., Burnashev, N., Monyer, H., Seeburg, P. H. \& Sakmann, B. Structural determinants of ion flow through recombinant glutamate receptor channels. Science 252, 1715-1718 (1991).
18. Oh, M. C. \& Derkach, V. A. Dominant role of the GluR2 subunit in regulation of AMPA receptors by CaMKII. Nat. Neurosci. 8, 853-854 (2005).

19. Traynelis, S. F., Silver, R. A. \& Cull-Candy, S. G. Estimated conductance of glutamate receptor channels activated during EPSCs at the cerebellar mossy fiber-granule cell synapse. Neuron 11, 279-289 (1993).

20. Benke, T. A. et al. Mathematical modelling of non-stationary fluctuation analysis for studying channel properties of synaptic AMPA receptors. J. Physiol. 537, 407-420 (2001).

21. Benke, T. \& Traynelis, S. F. AMPA-type glutamate receptor conductance changes and plasticity: still a lot of noise. Neurochem. Res. 94, 713-10 (2018).

22. Palmer, M. J., Isaac, J. T. R. \& Collingridge, G. L. Multiple, developmentally regulated expression mechanisms of long-term potentiation at CA1 synapses. J. Neurosci. 24, 4903-4911 (2004).

23. Lüthi, A. et al. Bi-directional modulation of AMPA receptor unitary conductance by synaptic activity. BMC Neurosci. 5, 44 (2004).

24. Andrásfalvy, B. K. \& Magee, J. C. Changes in AMPA receptor currents following LTP induction on rat CA1 pyramidal neurones. J. Physiol. 559, 543-554 (2004).

25. Guire, E. S., Oh, M. C., Soderling, T. R. \& Derkach, V. A. Recruitment of calcium-permeable AMPA receptors during synaptic potentiation is regulated by CaM-kinase I. J. Neurosci. 28, 6000-6009 (2008).

26. Park, P. et al. Calcium-permeable AMPA receptors mediate the induction of the protein kinase A-dependent component of long-term potentiation in the hippocampus. J. Neurosci. 36, 622-631 (2016).

27. Park, P. et al. The role of calcium-permeable AMPARs in long-term potentiation at principal neurons in the rodent hippocampus. Front. Synaptic Neurosci. 10, 42 (2018).

28. Geiger, J. R. et al. Relative abundance of subunit mRNAs determines gating and $\mathrm{Ca}^{2+}$ permeability of AMPA receptors in principal neurons and interneurons in rat CNS. Neuron 15, 193-204 (1995).

29. Barad, M., Bourtchouladze, R., Winder, D. G., Golan, H. \& Kandel, E. Rolipram, a type IV-specific phosphodiesterase inhibitor, facilitates the establishment of long-lasting long-term potentiation and improves memory. Proc. Natl Acad. Sci. 95, 15020-15025 (1998).

30. Zhang, M. et al. Adenylyl cyclase anchoring by a kinase anchor protein AKAP5 (AKAP79/150) is important for postsynaptic $\beta$-adrenergic signaling. J. Biol. Chem. 288, 17918-17931 (2013).

31. Whitehead, G. et al. Acute stress causes rapid synaptic insertion of $\mathrm{Ca}^{2+}$ -permeable AMPA receptors to facilitate long-term potentiation in the hippocampus. Brain 136, 3753-3765 (2013).

32. Sanderson, J. L., Gorski, J. A. \& Dell'Acqua, M. L. NMDA receptor-dependen LTD requires transient synaptic incorporation of $\mathrm{Ca}^{2+}$-permeable AMPARs mediated by AKAP150-anchored PKA and calcineurin. Neuron 89, 1000-1015 (2016)

33. McGlade-McCulloh, E., Yamamoto, H., Tan, S.-E., Brickey, D. A. \& Soderling, T. R. Phosphorylation and regulation of glutamate receptors by calcium/ calmodulin-dependent protein kinase II. Nature 362, 640-642 (1993).

34. Lledo, P. M. et al. Calcium/calmodulin-dependent kinase II and long-term potentiation enhance synaptic transmission by the same mechanism. Proc. Natl Acad. Sci. USA 92, 11175-11179 (1995).

35. Huang, Y. Y. \& Kandel, E. R. Recruitment of long-lasting and protein kinase A-dependent long-term potentiation in the CA1 region of hippocampus requires repeated tetanization. Learn. Mem. 1, 74-82 (1994).

36. Bliss, T. V. P., Collingridge, G. L., Morris, R. G. M. \& Reymann, K. G. Long term potentiation in the hippocampus: discovery, mechanisms and function. Neuroforum 24, A103-A12018 (2018).

37. Bortolotto, Z. A. \& Collingridge, G. L. A role for protein kinase C in a form of metaplasticity that regulates the induction of long-term potentiation at CA1 synapses of the adult rat hippocampus. Eur. J. Neurosci. 12, 4055-4062 (2000).

38. Woo, N. H., Duffy, S. N., Abel, T. \& Nguyen, P. V. Temporal spacing of synaptic stimulation critically modulates the dependence of LTP on cyclic AMP-dependent protein kinase. Hippocampus 13, 293-300 (2003).

39. Park, P. et al. NMDA receptor-dependent long-term potentiation comprises a family of temporally overlapping forms of synaptic plasticity that are induced by different patterns of stimulation. Philos. Trans. R. Soc. B 369, 20130131-20130131 (2014)

40. Hayashi, Y. et al. Driving AMPA receptors into synapses by LTP and CaMKII: requirement for GluR1 and PDZ domain interaction. Science 287, 2262-2267 (2000).

41. Oh, M. C., Derkach, V. A., Guire, E. S. \& Soderling, T. R. Extrasynaptic membrane trafficking regulated by GluR1 serine 845 phosphorylation primes AMPA receptors for long-term potentiation. J. Biol. Chem. 281, 752-758 (2006)

42. Gao, C., Sun, X. \& Wolf, M. E. Activation of D1 dopamine receptors increases surface expression of AMPA receptors and facilitates their synaptic incorporation in cultured hippocampal neurons. J. Neurochem. 98, 1664-1677 (2006).

43. Plant, K. et al. Transient incorporation of native GluR2-lacking AMPA receptors during hippocampal long-term potentiation. Nat. Neurosci. 9, 602-604 (2006). 
44. Adesnik, H. \& Nicoll, R. A. Conservation of glutamate receptor 2-containing AMPA receptors during long-term potentiation. J. Neurosci. 27, 4598-4602 (2007).

45. Gray, E. E., Fink, A. E., Sarinana, J., Vissel, B. \& O’Dell, T. J. Long-term potentiation in the hippocampal CA1 region does not require insertion and activation of GluR2-lacking AMPA receptors. J. Neurophysiol. 98, 2488-2492 (2007).

46. Lu, Y. et al. Age-dependent requirement of AKAP150-anchored PKA and GluR2-lacking AMPA receptors in LTP. EMBO J. 26, 4879-4890 (2007).

47. Cizeron, M. et al. A brainwide atlas of synapses across the mouse life span. Science 369, 270-275 (2020).

48. Zhou, Z. et al. The C-terminal tails of endogenous GluA1 and GluA2 differentially contribute to hippocampal synaptic plasticity and learning. Nat. Neurosci. 21, 50-62 (2018).

49. Diering, G. H. \& Huganir, R. L. The AMPA receptor code of synaptic plasticity. Neuron 100, 314-329 (2018).

50. He, K. et al. Stabilization of $\mathrm{Ca}^{2+}$-permeable AMPA receptors at perisynaptic sites by GluR1-S845 phosphorylation. Proc. Natl Acad. Sci. 106, 20033-20038 (2009).

51. Hell, J. W. How Ca2+-permeable AMPA receptors, the kinase PKA, and the phosphatase PP2B are intertwined in synaptic LTP and LTD. Sci. Signal. 9, pe2 (2016).

52. Gardner, S. M. et al. Calcium-permeable AMPA receptor plasticity is mediated by subunit-specific interactions with PICK1 and NSF. Neuron 45, 903-915 (2005).

53. Jaafari, N., Henley, J. M. \& Hanley, J. G. PICK1 mediates transient synaptic expression of GluA2-lacking AMPA receptors during glycine-induced AMPA receptor trafficking. J. Neurosci. 32, 11618-11630 (2012).

54. Terashima, A. et al. Regulation of synaptic strength and AMPA receptor subunit composition by PICK1. J. Neurosci. 24, 5381-5390 (2004).

55. Morita, D., Rah, J.-C. \& Isaac, J. T. R. Incorporation of inwardly rectifying AMPA receptors at silent synapses during hippocampal long-term potentiation. Philos. Trans. R. Soc. B 369, 20130156 (2014).

56. Liu, S. Q. \& Cull-Candy, S. G. Synaptic activity at calcium-permeable AMPA receptors induces a switch in receptor subtype. Nature 405, 454-458 (2000).

57. Noh, K.-M. et al. Blockade of calcium-permeable AMPA receptors protects hippocampal neurons against global ischemia-induced death. Proc. Natl Acad. Sci. USA 102, 12230-12235 (2005).

58. Isaac, J. T., Nicoll, R. A. \& Malenka, R. C. Evidence for silent synapses: implications for the expression of LTP. Neuron 15, 427-434 (1995).

59. Liao, D., Hessler, N. A. \& Malinow, R. Activation of postsynaptically silent synapses during pairing-induced LTP in CA1 region of hippocampal slice. Nature 375, 400-404 (1995).

60. Nonaka, M. et al. Everyday memory: towards a translationally effective method of modelling the encoding, forgetting and enhancement of memory. Eur. J. Neurosci. 46, 1937-1953 (2017).

61. $\mathrm{Hu}, \mathrm{H}$. et al. Emotion enhances learning via norepinephrine regulation of AMPA-receptor trafficking. Cell 131, 160-173 (2007).

62. Takeuchi, T. et al. Locus coeruleus and dopaminergic consolidation of everyday memory. Nature 537, 357-362 (2016).

63. Anderson, W. W. \& Collingridge, G. L. Capabilities of the WinLTP data acquisition program extending beyond basic LTP experimental functions. $J$. Neurosci. Methods 162, 346-356 (2007).
64. Incontro, S., Asensio, C. S., Edwards, R. H. \& Nicoll, R. A. Efficient, complete deletion of synaptic proteins using CRISPR. Neuron 83, 1051-1057 (2014)

65. Sanjana, N. E., Shalem, O. \& Zhang, F. Improved vectors and genome-wide libraries for CRISPR screening. Nat. Methods 11, 783-784 (2014).

\section{Acknowledgements}

This work was supported by the MRC, ERC, CIHR Foundation Grant \#154276 (G.L.C.) and Brain Canada Foundation (G.L.C.) through the Canada Brain Research Fund, with the financial support of Health Canada. G.L.C. is the holder of the Krembil Family Chair in Alzheimer's Research. This work was also supported by The National Honor Scientist Program (NRF-2012R1A3A1050385) of Korea (B.-K.K.)

\section{Author contributions}

P.P. and G.L.C. conceived the study. P.P. carried out the experimental work. K.-H.K. and J.-i. K. helped with CRISPR experiments. T.M.S., Z.A.B., H.K., M.Z. and B.-K.K. provided advice and technical and/or financial support. P.P., J.G., C.A.B. and G.L.C. wrote the manuscript.

\section{Competing interests}

The authors declare no competing interests.

\section{Additional information}

Supplementary information is available for this paper at https://doi.org/10.1038/s41467020-20523-3.

Correspondence and requests for materials should be addressed to G.L.C.

Peer review information Nature Communications thanks Graham Diering, Johannes Hell, and other, anonymous, reviewers for their contributions to the peer review of this work. Peer review reports are available.

Reprints and permission information is available at http://www.nature.com/reprints

Publisher's note Springer Nature remains neutral with regard to jurisdictional claims in published maps and institutional affiliations.

Open Access This article is licensed under a Creative Commons Attribution 4.0 International License, which permits use, sharing, adaptation, distribution and reproduction in any medium or format, as long as you give appropriate credit to the original author(s) and the source, provide a link to the Creative Commons license, and indicate if changes were made. The images or other third party material in this article are included in the article's Creative Commons license, unless indicated otherwise in a credit line to the material. If material is not included in the article's Creative Commons license and your intended use is not permitted by statutory regulation or exceeds the permitted use, you will need to obtain permission directly from the copyright holder. To view a copy of this license, visit http://creativecommons.org/ licenses/by/4.0/.

(C) The Author(s) 2021 